\title{
Influence of Data Irregularities on 3D Common Offset Migration
}

\author{
F. Assouline ${ }^{1,2}$ and P. Lailly ${ }^{1}$ \\ 1 Institut français du pétrole, 1 et 4, avenue de Bois-Préau, 92852 Rueil-Malmaison Cedex - France \\ 2 Université de Pau et des Pays de l'Adour, avenue de l'Université, 64012 Pau - France \\ e-mail: frederic_assouline@yahoo.fr - patrick.lailly@ifp.fr
}

\begin{abstract}
Résumé - Influence d'irrégularités dans l'échantillonnage des données sur la migration 3D par déport - En sismique 3D, le déport est un vecteur caractérisé par la distance source-récepteur et par un azimut. Les irrégularités d'acquisition (dérive de la flûte en sismique marine par exemple) font que l'on ne peut véritablement disposer de données à déport constant et que, en conséquence, la notion même de migration par déport perd tout sens. Il convient alors d'introduire la notion de migration par classe de déports, qui constitue une extension naturelle de la migration à déport commun : dans cette extension, on migre toutes les traces sismiques appartenant à une même classe de déports, c'est-à-dire toutes les traces dont le déport est proche d'un déport donné. Le résultat dépendra, bien sûr, de la façon dont la classe de déports aura été constituée ou, en d'autres termes, de ce que l'on entendra par « proche d'un déport donné ».

Dans cet article, nous regardons, par le biais d'une étude numérique 3D, comment les classes de déports doivent être construites si l'on veut que les images migrées soient dépourvues d'artéfacts susceptibles d'en perturber l'interprétation (c'est sur cette interprétation que repose le succès des techniques d'analyse de vitesse de migration). La construction de telles classes de déports revient à trouver un compromis entre :

- des classes de déports trop lâches, c'est-à-dire dans lesquelles des déports très différents (et notamment des azimuts très différents) se trouveraient mélangés ;

- des classes de déports trop étroites, qui conduiraient à des traces sismiques très éparses avec, en conséquence, une illumination inadéquate des réflecteurs.

La qualité des images migrées se trouve principalement affectée par deux paramètres caractéristiques de la classe de déports : la régularité des variations du déport et la distribution des points milieux. La migration par classe de déports apparaît comme une procédure robuste tant que, à l'intérieur d'une même classe, le déport varie lentement en fonction du point milieu. Enfin, les artéfacts résultant du mixage des azimuts sont d'autant moins importants que la distance moyenne source-récepteur est, à l'intérieur de la classe de déports considérée, petite.
\end{abstract}

Mots-clés : sismique réflexion, acquisition sismique 3D, migration $3 \mathrm{D}$ avant sommation.

\footnotetext{
Abstract - Influence of Data Irregularities on 3D Common Offset Migration - In 3D seismics offset is a vector characterized by the source-receiver distance and azimuth. Due to irregularities in the acquisition (for instance streamer feathering in marine acquisition) common offset data are not available and, in turn, 3D common offset migration does not make sense any longer. We then introduce the concept of migration by offset class: it is a straightforward extension of common offset migration in which we migrate all traces that belong to a given offset class, namely all traces with offset close to a given one. The result of such a migration depends of course on how the offset class is constructed or, in other words, on what is meant with "close".
} 
The aim of this paper is to investigate by means of a 3D numerical study how to design offset classes so as to obtain migrated images that can be interpreted without trouble (such an interpretation is a key element for a successful migration velocity analysis). We have to find a trade-off between two extremes:

- a too loose offset class that would mix heterogeneous offsets (in particular mixing substantially different azimuths can be dangerous);

- a too narrow offset class that would result in sparse seismic traces with, in turn, an inadequate coverage of the reflector.

The regularity, within an offset class, of the offset variation as well as the one of the distribution of midpoints are parameters that can have a drastic influence on the quality of the migrated image. It is shown that migration by offset class appears as a robust procedure all the more than the offset varies slowly with the midpoint coordinate within an offset class. Besides, the smaller the mean offset norm, the lower is the contamination of the image due to the mixing of azimuths within an offset class.

Keywords: reflection seismology, 3D seismic acquisition, 3D prestack migration.

\section{INTRODUCTION}

For the seismic imaging of complex geological structures (i.e. structures involving strong lateral velocity variations) the KIM's consortium (Kinematic Inversion Methods) proposes the SMART (Sequential Migration Aided Reflection Tomography) approach (Lailly and Ehinger, 1991; Ehinger and Lailly, 1995). This migration velocity analysis technique uses a detour through the prestack depth migrated domain for accessing multi-offset kinematical information hardly accessible in the time domain.

In $3 \mathrm{D}$, such a method, as well as all other migration velocity analysis methods, relies on a sound interpretation of 3D offset migrated images. However, because of irregularities in seismic data acquisition (e.g. streamer feathering), seismic traces associated with a given offset are very few in general: by offset we mean here (and all along this paper) the vector characterized by the source-receiver distance and azimuth. As a consequence, a genuine common offset migrated image would involve important artifacts resulting from an important lack of illumination.

The goal of this paper is to determine how to produce nice (i.e. well suited for an interpretation) migrated images in the case of irregularities in the seismic acquisition.

\section{COMMON OFFSET MIGRATION}

In a 3D situation, any source-receiver pair can be identified by an offset and a midpoint, which are defined by:

$$
\begin{aligned}
& \vec{h}_{0}=\vec{r}-\vec{s} \\
& \vec{q}=\frac{\vec{s}+\vec{r}}{2}
\end{aligned}
$$

where vectors $\vec{s}$ and $\vec{r}$ characterize the locations of the source and the receiver, respectively.

For a given velocity field $v$, the migrated image at a subsurface point $M$ for offset $\vec{h}_{0}$ is defined by superposition of elementary migrated images $m_{e l}^{v}$ associated with all source-receiver pairs $(S, R)$ with offset $\vec{h}_{0}$. We consider in this paper the simplest superposition, namely the diffraction stack formula (Schneider, 1978):

$$
m_{\vec{h}_{0}}^{v}(M)=\sum_{\vec{q}} m_{e l}^{v}\left(M ; \vec{q}-\frac{\vec{h}_{0}}{2}, \vec{q}+\frac{\vec{h}_{0}}{2}\right)
$$

where the elementary migrated image is defined as:

$$
\begin{aligned}
& m_{e l}^{v}\left(M ; \vec{q}-\frac{\vec{h}_{0}}{2}, \vec{q}+\frac{\vec{h}_{0}}{2}\right)= \\
& d\left(\vec{q}-\frac{\vec{h}_{0}}{2}, \vec{q}+\frac{\vec{h}_{0}}{2}, t_{m}\left(M ; \vec{q}-\frac{\vec{h}_{0}}{2}, \vec{q}+\frac{\vec{h}_{0}}{2}, v\right)\right) \\
& d\left(\vec{q}-\frac{\vec{h}_{0}}{2}, \vec{q}+\frac{\vec{h}_{0}}{2}, t\right)
\end{aligned}
$$

where

is the seismic trace associated with the source receiver pair $(S, R)$ and $t_{m}$ is the traveltime from $S$ to $M$ to $R$ in the velocity field $v$.

Note that, if the midpoints are adequately sampled, Equation (3) can be viewed as a discrete version of integral:

$$
m_{\vec{h}_{0}}^{v}(M)=\iint_{q_{x} q_{y}} m_{e l}^{v}\left(M ; \vec{q}-\frac{\vec{h}_{0}}{2}, \vec{q}+\frac{\vec{h}_{0}}{2}\right) \mathrm{d} q_{x} \mathrm{~d} q_{y}
$$

where $\left(q_{x}, q_{y}\right)$ are the midpoint coordinates.

Considering an event with a single arrival time $t_{e}(\vec{s}, \vec{r})$, use of the stationary phase method (see for instance Bleistein, 1984) yields the location of the support of the offset migrated event by the following system:

$$
\left\{\begin{array}{r}
t_{m}\left(M ; \vec{s}, \vec{s}+\vec{h}_{0}, v\right)-t_{e}\left(\vec{s}, \vec{s}+\vec{h}_{0}\right)=0 \\
\quad \text { (zero phase condition) } \\
\frac{\partial}{\partial s_{i}}\left[t_{m}\left(M ; \vec{s}, \vec{s}+\vec{h}_{0}, v\right)-t_{e}\left(\vec{s}, \vec{s}+\vec{h}_{0}\right)\right]=0 \quad i=x, y \\
\text { (stationary phase condition) }
\end{array}\right.
$$

where $\left(s_{x}, s_{y}\right)$ are the source coordinates. 
(a)
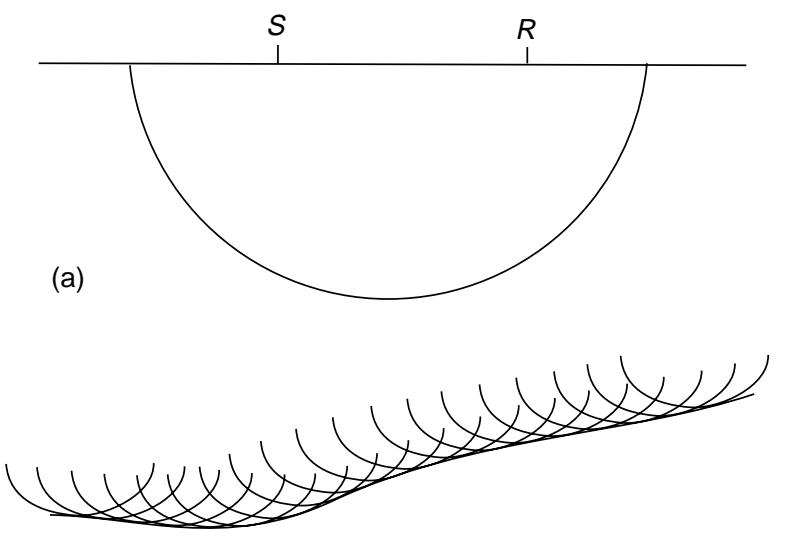

(b)

Figure 1

Construction of the offset migrated event in 2D.

(a) The traveltime $t_{e}\left(s, s+h_{0}\right)$ defines the isochron, which is the ellipse (for a homogeneous velocity field) with focal points $S$ and $R$.

(b) The geometry of the migrated event is the envelope of the isochrons associated with each source-receiver pair.

This mathematical result can be interpreted as follows: for a given source-receiver pair, the first equation defines an isochron surface, i.e. a set of points $M$ which are possible diffraction points (for a homogeneous velocity field, an isochron is an ellipsoid). Together with the second equation, System (6) defines the geometry of a migrated event as the envelope of the family, parameterized by $\vec{s}$, of isochrons. This holds as well for the exact as for an erroneous migration velocity field (Figs. la and $1 b$ ).

This theory can be experimentally confirmed by running 3D common offset migrations, with different migration velocities, on 3D regular synthetic data. For this experiment, the 3D two-layer model described in Figures $2 \mathrm{a}$ and $2 \mathrm{~b}$ has been created: the interface shows a relatively high curvature and extends from -3 to $21 \mathrm{~km}$ in the $x$ direction, -4 to $16 \mathrm{~km}$ in the $y$ direction. The interface, explicit in the $z$ direction, is represented by $60 \times 40 \mathrm{~B}$-spline parameters. Above the interface the velocity field is constant: $v=3.0 \mathrm{~km} / \mathrm{s}$.

The survey involves regularly distributed midpoints with a $50 \mathrm{~m}$ spacing both in the $x$ and $y$ directions, a fixed azimuth $\alpha=0$ ( $\alpha$ being the angle between the direction of the offset and the $x$ axis) and a constant source-receiver distance $\left\|\vec{h}_{0}\right\|=$ $3000 \mathrm{~m}$ (source locations are displayed in Figure 3a) ${ }^{1}$. The seismic traces are generated by our two-point ray tracing software Jerry (Jurado et al., 1996) which provides quite

(1) The choice to lead the study principally with a large offset norm has been made because, as it is shown in Figure 17 for instance, the contamination of the image due to offset fluctuations is stronger for large than for small offset norms. accurate reflection traveltimes when the velocity is constant. These times are then convolved by a seismic wavelet, a Ricker centered on $25 \mathrm{~Hz}$, so as to produce seismic traces. We have computed the migrated data (with a classical diffraction stack algorithm) on a cube which extends from 3.5 to $11 \mathrm{~km}$ in the $x$ direction and from 4 to $6 \mathrm{~km}$ in the $y$ direction.

For the exact velocity, as predicted by the theory, the migrated event is the one that peaks on the reflector of the model (Figs. $4 a$ and $4 b$ ). There are also artifacts often referred to migration smiles (events 1 and 2) and which are due to the finite extent of the acquisition, i.e. data truncation. Indeed the migrated image, which is defined by Equation (3) as the envelope of the isochrons associated with each sourcereceiver pair, is made by constructive interferences between elementary migrated images everywhere except where there is a discontinuity in the data. Other artifacts (event 3 ) are due to insufficient spatial sampling that results from a locally too low density of midpoints in the survey.

To save computing time, we will, in the sequel, restrict the imaging to a domain that ranges from 6 to $8.3 \mathrm{~km}$ in the $x$ direction, from 4 to $6 \mathrm{~km}$ in the $y$ direction and whose extension in the $z$ direction is $1 \mathrm{~km}$ on either side of the reflector (Fig. 5). Such a domain indeed allows:

- to show how the steep slope of the anticline is imaged;

- to see (thanks to the large window in the $z$ direction) the migrated event even with a quite erroneous migration velocity;

while avoiding artifacts caused by holes in the illumination (Fig. 3b), at least for the exact migration velocity.

For a $33 \%$ too low migration velocity $(v=2000 \mathrm{~m} / \mathrm{s})$ the migrated event is not as deep as the one obtained with the exact velocity and its slope is not as steep (Fig. 6).

For a $33 \%$ too high migration velocity $(v=4000 \mathrm{~m} / \mathrm{s})$ the geometry of the migrated event is totally modified and three cusp branches appear (Fig. 7). There are also branches due to data truncation: use of this migration velocity makes the migrated event move on the left in the $x$ direction as compared to the result obtained with the exact velocity.

\section{OFFSET CLASS MIGRATION}

In practice data associated with a given offset $\vec{h}_{0}$ consist of very sparse seismic traces and, in turn, a common offset migration becomes inadequate. This leads us to extend the notion of common offset migration by introducing offset class migration. We define an offset class $C_{\vec{h}_{0}}$ as the set of all source-receiver pairs such that their offset $\vec{h}=\vec{r}-\vec{s}$ belongs to a neighborhood of vector $\vec{h}_{0}$. The offset class migrated image at a subsurface point $M$ is then defined as previously by 

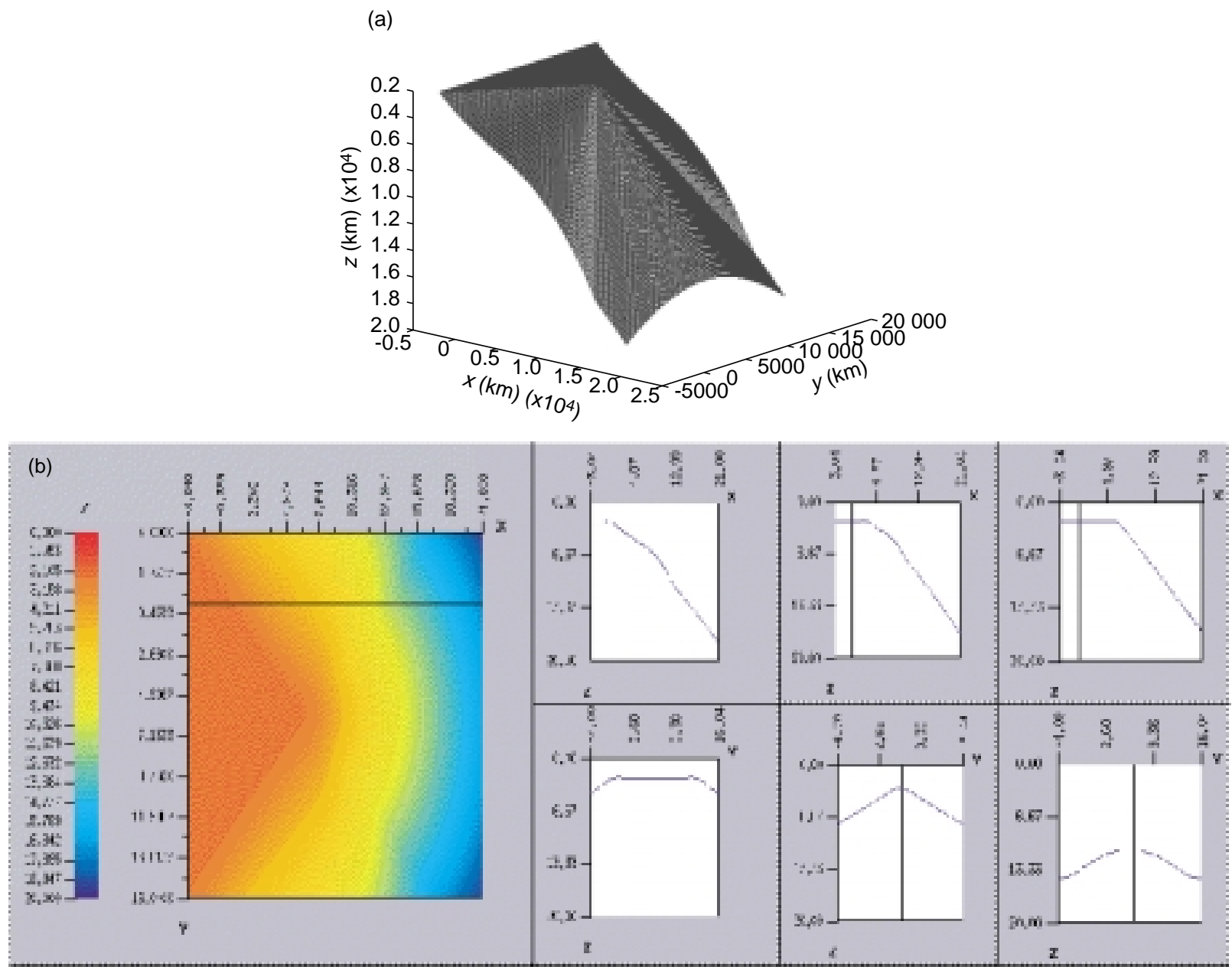

Figure 2

The synthetic 3D model. The velocity field above the reflector is constant, $v=3.0 \mathrm{~km} / \mathrm{s}$.

(a) A 3D view of the interface.

(b) Map of the interface and different sections in the model:

$y$ sections (top): from left to right: $y=2 \mathrm{~km}, y=4 \mathrm{~km}, y=6 \mathrm{~km} ; x$ sections (bottom): from left to right: $x=1 \mathrm{~km}, x=7 \mathrm{~km}, x=17 \mathrm{~km}$.

superposition of elementary migrated images associated with all seismic traces of the considered offset class:

$$
m_{C_{\vec{h}_{0}}}^{v}(M)=\sum_{\vec{q} \in C_{\vec{h}_{0}}} m_{e l}^{v}\left(M ; \vec{q}-\frac{\vec{h}}{2}, \vec{q}+\frac{\vec{h}}{2}\right)
$$

We have used such an imaging technique on synthetic data generated as before (that is by using Jerry on the model in Figures 2a and 2b) but associated with source-receiver pairs whose locations come from a field marine acquisition in the North Sea. Such an acquisition involves weak variations in the azimuth.

We have considered far offset data: $\left\|\vec{h}_{0}\right\|=3000 \mathrm{~m}$. We have constituted the offset class consisting of source-receiver pairs whose distance belongs to [2950 m; $3050 \mathrm{~m}$ ] (Figs. $8 a$ and $8 b$ ).
The offset class migrated images (Fig. 9) show imaging artifacts strong enough to harm a migration velocity analysis.

A more selective offset class gathering source-receiver pairs whose distance belongs to [2995 m; $3005 \mathrm{~m}$ ] does not really help (Figs. 10a, 10b and 11).

At this stage we may wonder if offset class migration is a theoretically justified seismic imaging technique, or if we lack insights on how to constitute offset classes.

\section{THEORETICAL FOUNDATION OF OFFSET CLASS MIGRATION}

As common offset migration, the theoretical foundation of offset class migration is based on the analysis of a continuous problem. To generalize the continuous definition (5) to offset 
(a)

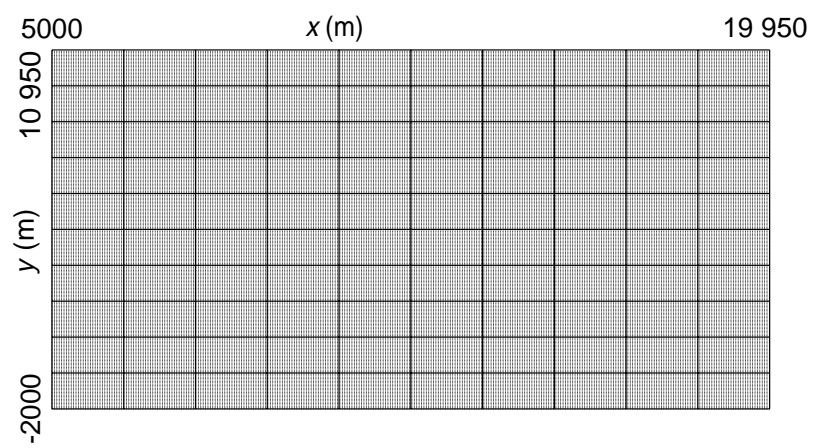

(b)

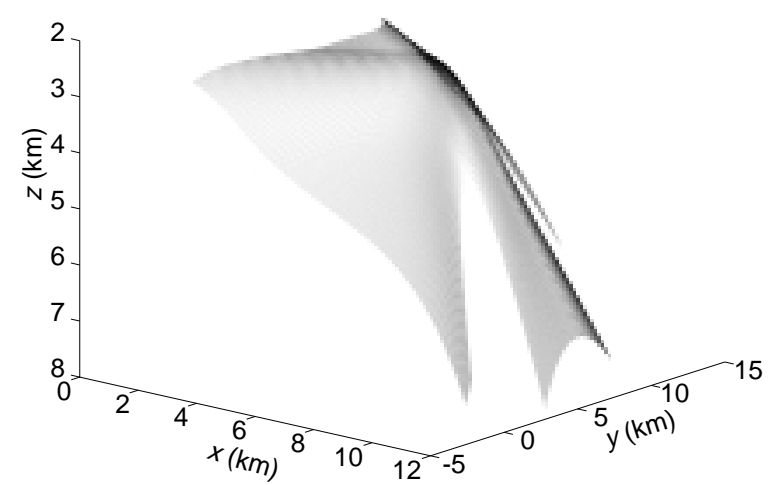

Figure 3

Source locations of a regular synthetic acquisition: the midpoints are regularly distributed with a spacing of $50 \mathrm{~m}$ both in the $x$ and $y$ directions, $\left\|\vec{h}_{0}\right\|=3000 \mathrm{~m}$ and $\alpha=0$.

(a) Map of source locations.

(b) $3 \mathrm{D}$ view of the impact points on the reflector.

class migration, we now consider offsets that depend on midpoint $\vec{q}$ : these offsets are defined by the function $\tilde{h}_{0}(\vec{q})$ that will be referred to as the offset class function and that is assumed to remain in the vicinity of $\vec{h}_{0}$ (see Figs. $12 a$ and $12 b$ for a $2 D$ illustration). This function thus defines a set of source-receiver pairs that depend on two parameters, the midpoint coordinates $q_{x}$ and $q_{y}$. Given an offset class function $\tilde{h}_{0}(\vec{q})$, we introduce the continuous definition of migration by offset class:

$$
m_{\tilde{h}_{0}}^{v}(M)=\iint_{q_{x} q_{y}} m_{e l}^{v}\left(M ; \vec{q}, \tilde{h}_{0}(\vec{q})\right) \mathrm{d} q_{x} \mathrm{~d} q_{y}
$$

which clearly appears as an extension of Equation (5).

Also, if the midpoints are adequately sampled, Formula (7) appears as a discrete approximation of Equation (8). In this context, the source-receiver pairs that contribute in the construction of the offset class migrated image appear as samples of the offset class function (see Figs. $12 a$ and $12 b$ for a $2 D$ illustration).

The analysis given in the Appendix proves the existence of offset class migrated events, even in the case of an erroneous migration velocity, provided that the offset class function is smooth (the variation of offset with midpoints coordinates must not show discontinuities). The geometry of the offset class migrated event appears as the envelope of isochrons associated with all source-receiver pairs defined by the offset class function. Additional results about the geometry of the offset class migrated events can be found in the Appendix.

With these results, offset class migration appears to rely on a sound theoretical basis.

\section{AN EXPERIMENTAL STUDY}

The theory outlined in the previous section says almost nothing on how to constitute offset classes for a good imaging (we just know that the offset class function has to be smooth). The difficulty met when we tried to image synthetic data associated with a field acquisition geometry should lie in incorrectly constituted offset classes. Indeed the offset classes that were constituted show:

- offset variations both in norm and in azimuth and we do not know to what extent such variations are allowed;

- a non-regular spacing between midpoints.

The goal of our experimental study is to investigate the influence of the parameters that define an offset class on the quality of the resulting migrated image. For this, two series of experiments have been carried out.

In the first one, the distribution of midpoints is uniform and either the norm is fixed (mixing of azimuths), or the azimuth is fixed (mixing of norms) (Figs. 13a and 13b). In the second series of experiments, we study the influence on the image of a non-uniform distribution of midpoints, for a fixed offset. The quality of the migrated image is essentially evaluated with a visual criterion. It is thus dependent on the mode of graphical representation that is chosen. In all the experiments below, the scaling factor is the same so as to allow comparison between results associated with various acquisitions but involving the same number of source-receiver pairs.

\subsection{Variation of Offset for a Uniform Distribution of Midpoints}

In this first part we consider offset classes involving varying offsets but regularly sampled midpoints (the midpoints lie on 


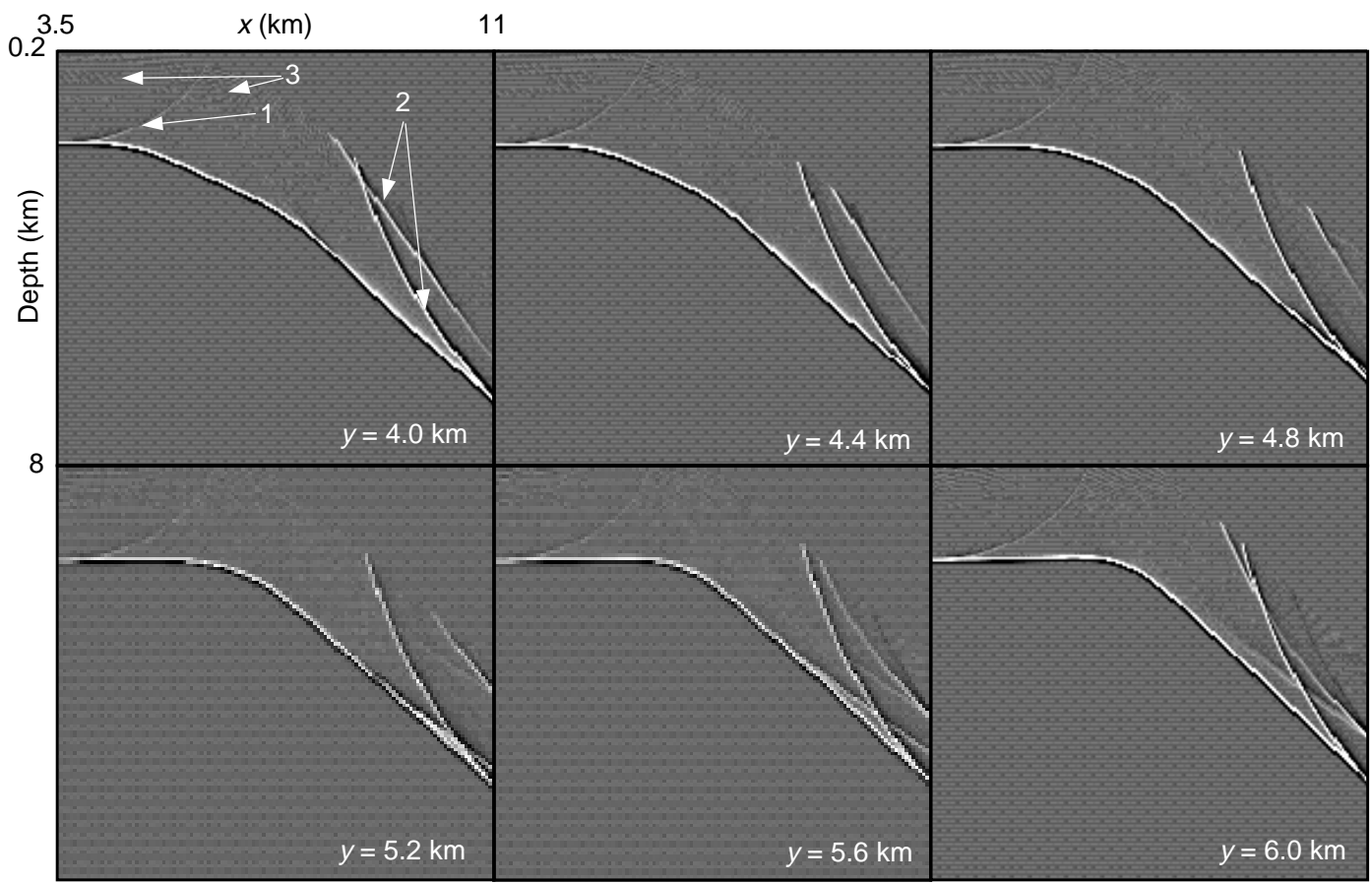

(a)

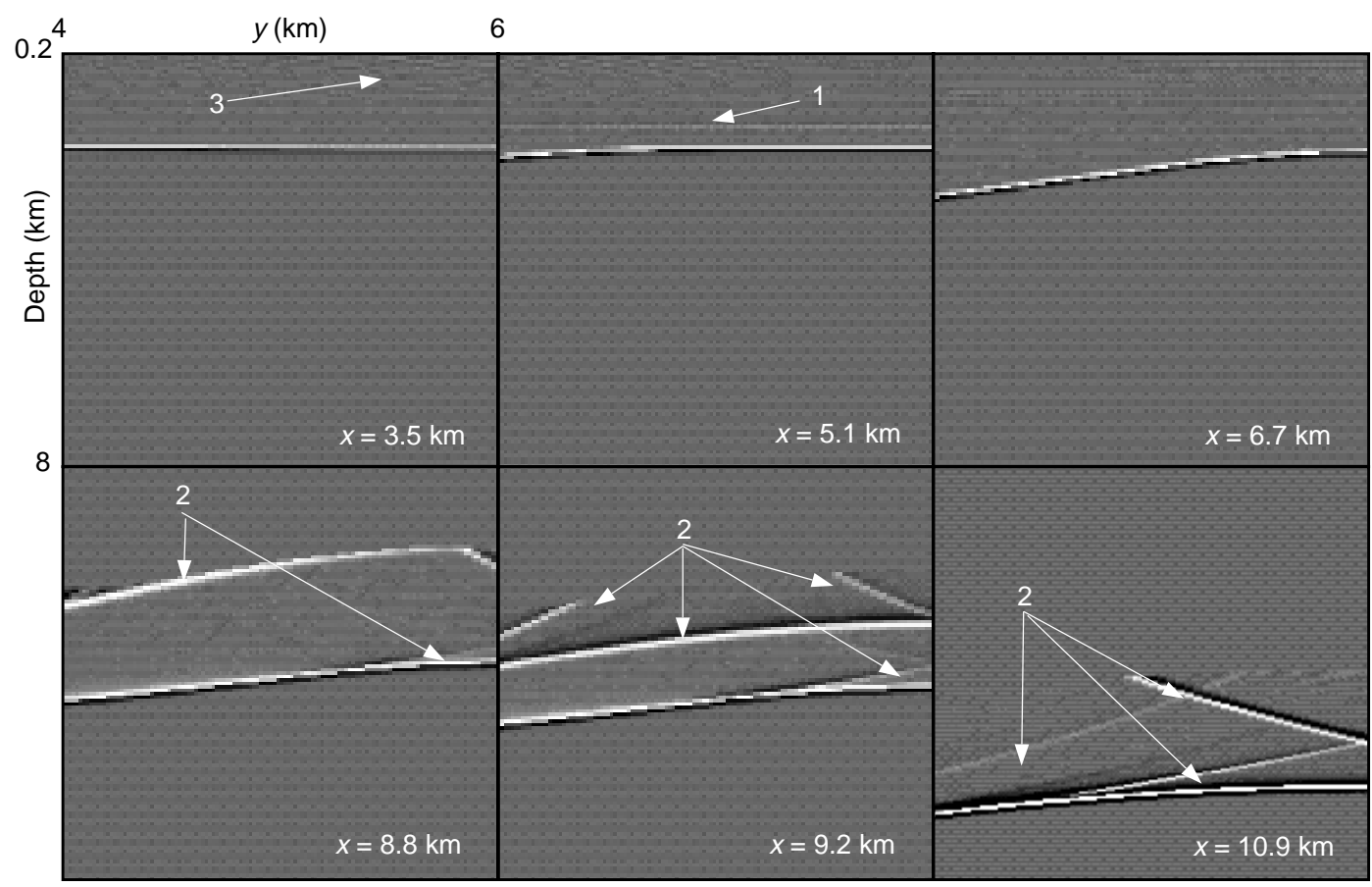

(b)

Figure 4

Cube of offset migrated images $\left(\left\|\vec{h}_{0}\right\|=3000 \mathrm{~m}\right)$ with the exact migration velocity $v=3000 \mathrm{~m} / \mathrm{s}$.

(a) Some $y$ sections in the cube.

(b) Some $x$ sections in the cube. Events 1 and 2 (called smiles) are artifacts of migration generated by data truncation due to the finite extent of the acquisition array in the $x$ and $y$ directions. Event 3 is due to insufficient spatial sampling that results from a locally too low density of midpoints in the survey. 


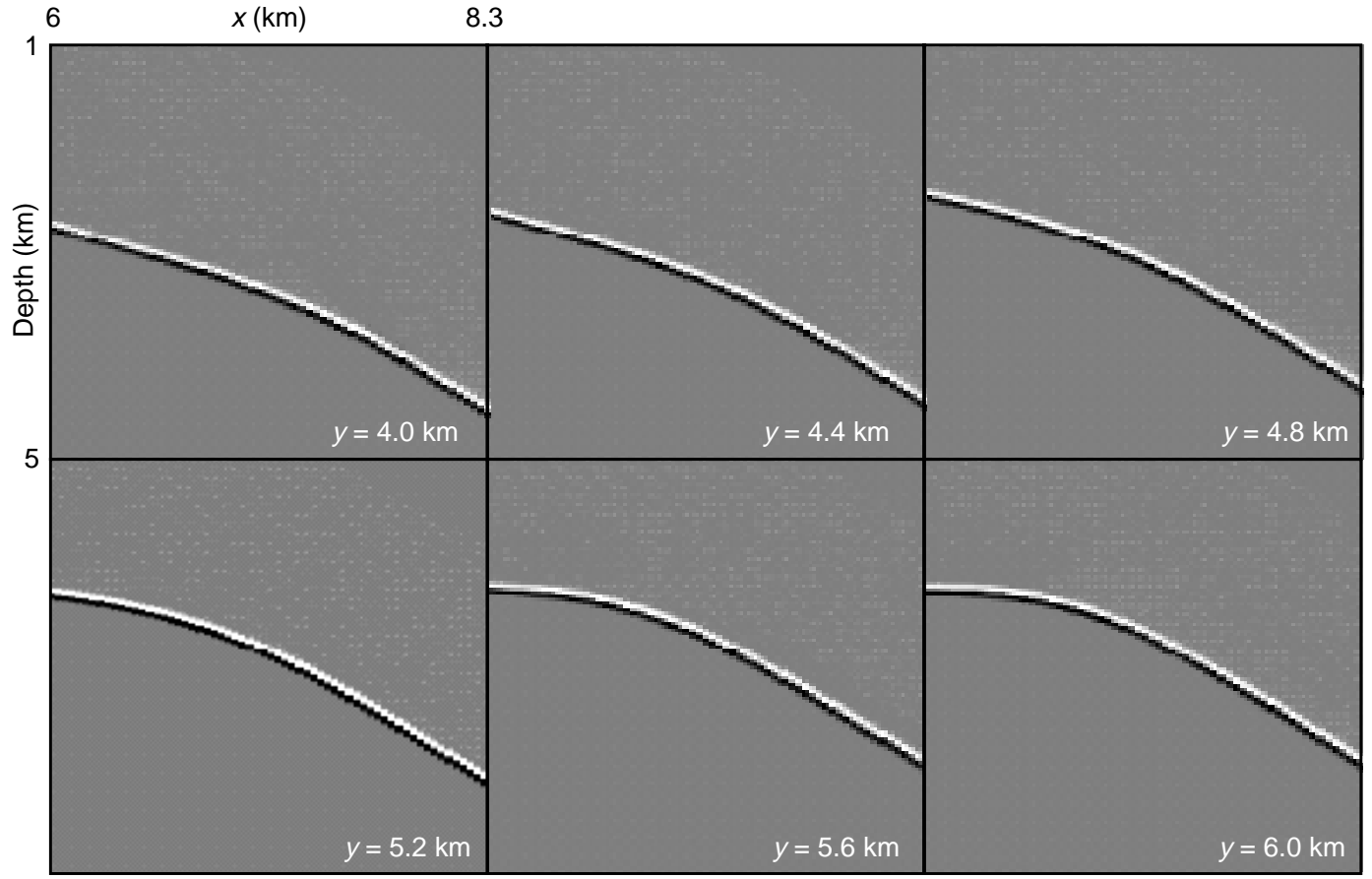

Figure 5

$y$ sections in the cube of offset migrated images restricted to $x \in[6 \mathrm{~km} ; 8.3 \mathrm{~km}]$ and $y \in[4 \mathrm{~km} ; 6 \mathrm{~km}]$ with $\left\|\vec{h}_{0}\right\|=3000 \mathrm{~m}$ and the exact migration velocity $v=3000 \mathrm{~m} / \mathrm{s}$.

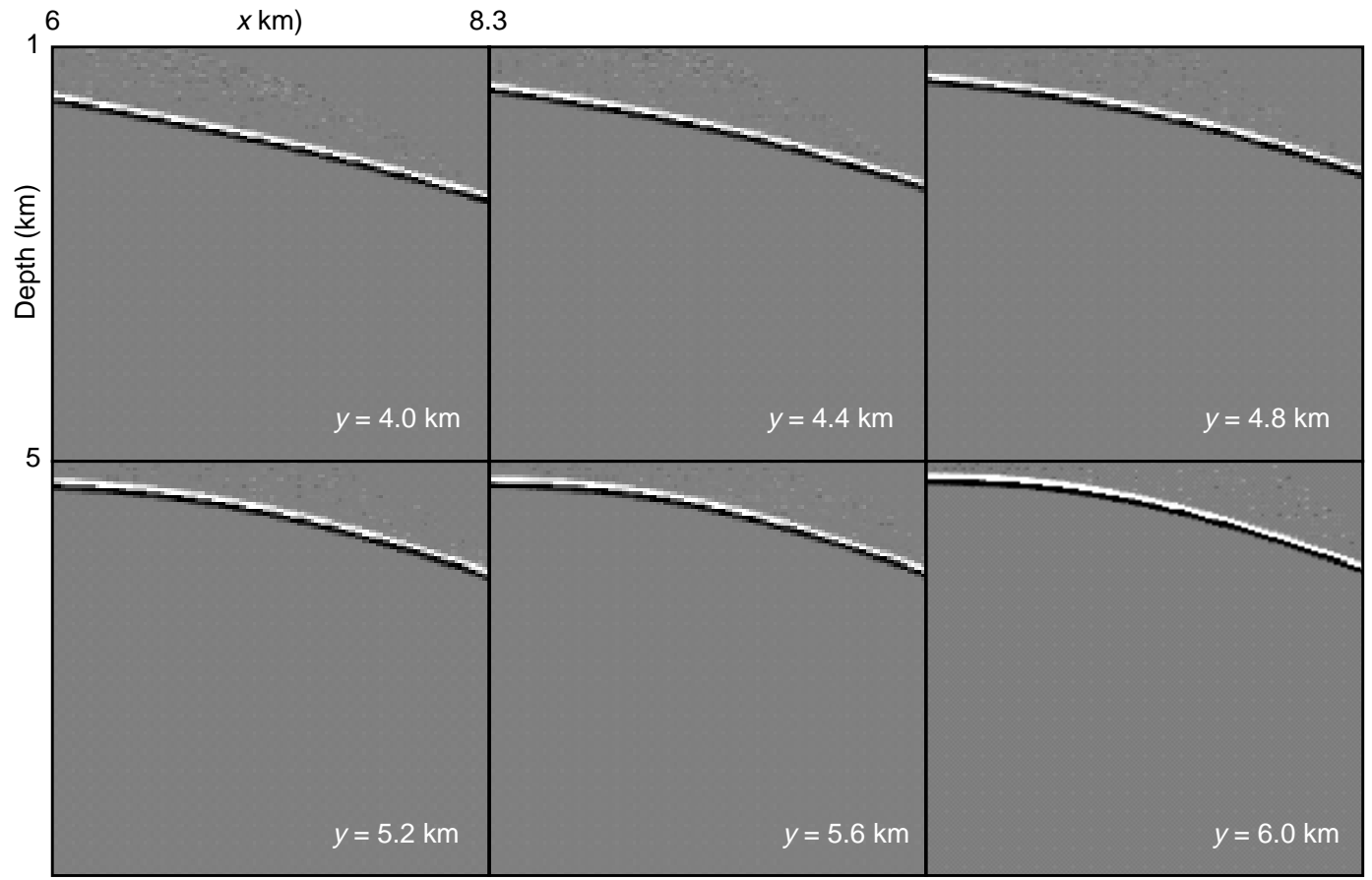

Figure 6

$y$ sections in the cube of offset migrated images with $\left\|\vec{h}_{0}\right\|=3000 \mathrm{~m}$ and a too low migration velocity $v=2000 \mathrm{~m} / \mathrm{s}$. 


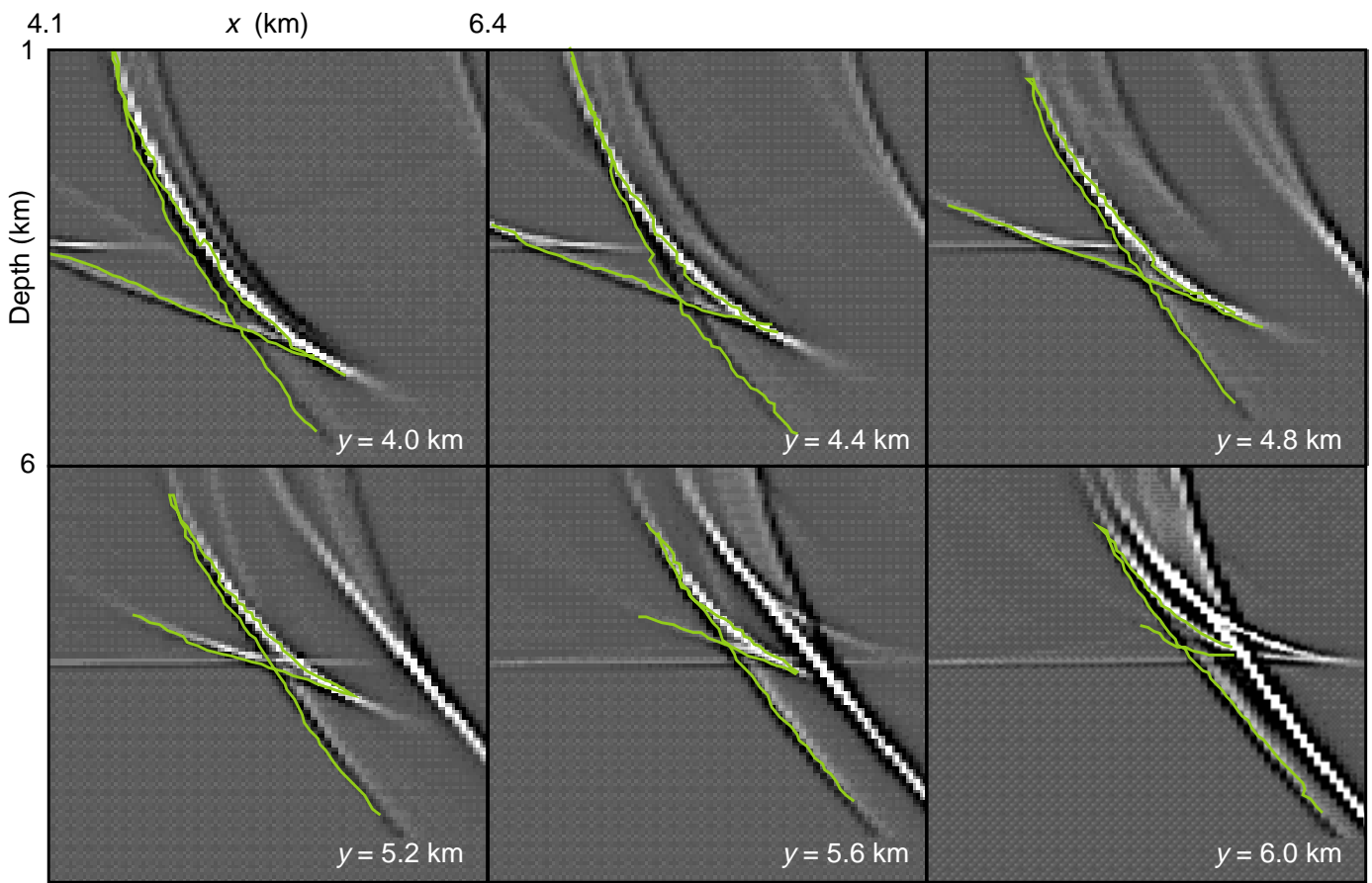

Figure 7

$y$ sections in the cube of offset migrated images with $\left\|\vec{h}_{0}\right\|=3000 \mathrm{~m}$ and a too high migration velocity $v=4000 \mathrm{~m} / \mathrm{s}$. The three cusp branches colored in green are the consequences of the erroneous migration velocity. Other events are the so-called migration smiles due to data truncation.

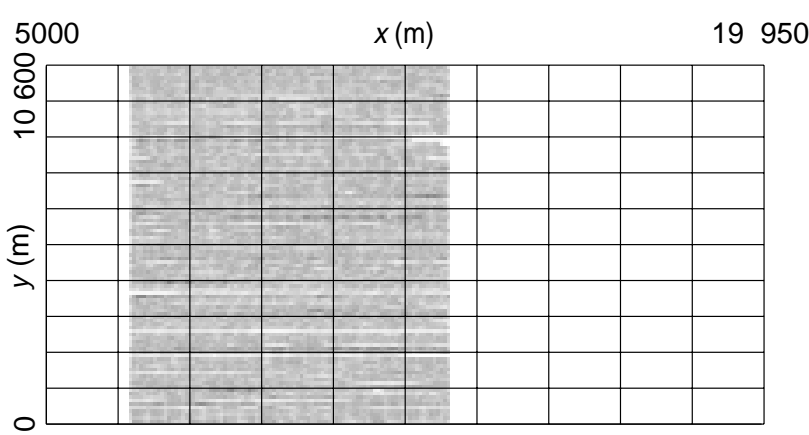

(a)

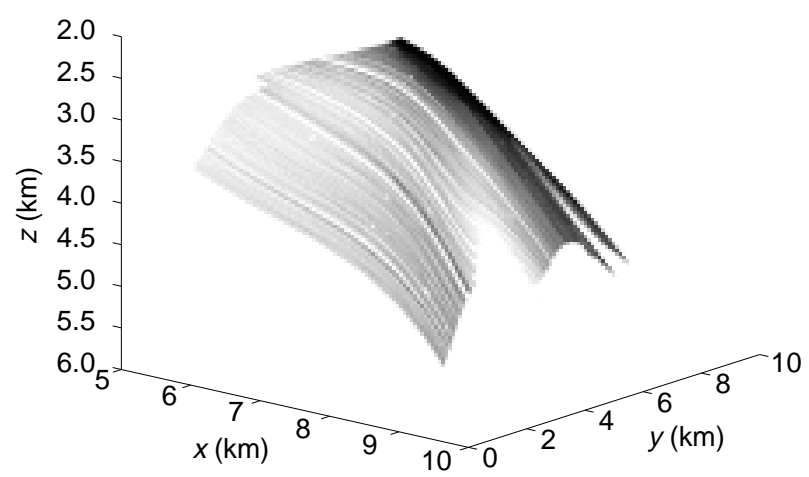

(b)

Figure 8

Survey from a marine acquisition in the North Sea.

(a) Map of locations of sources that have a receiver at a distance ranging within [2950 $\mathrm{m} ; 3050 \mathrm{~m}]$.

(b) $3 \mathrm{D}$ view of the impact points on the reflector. 


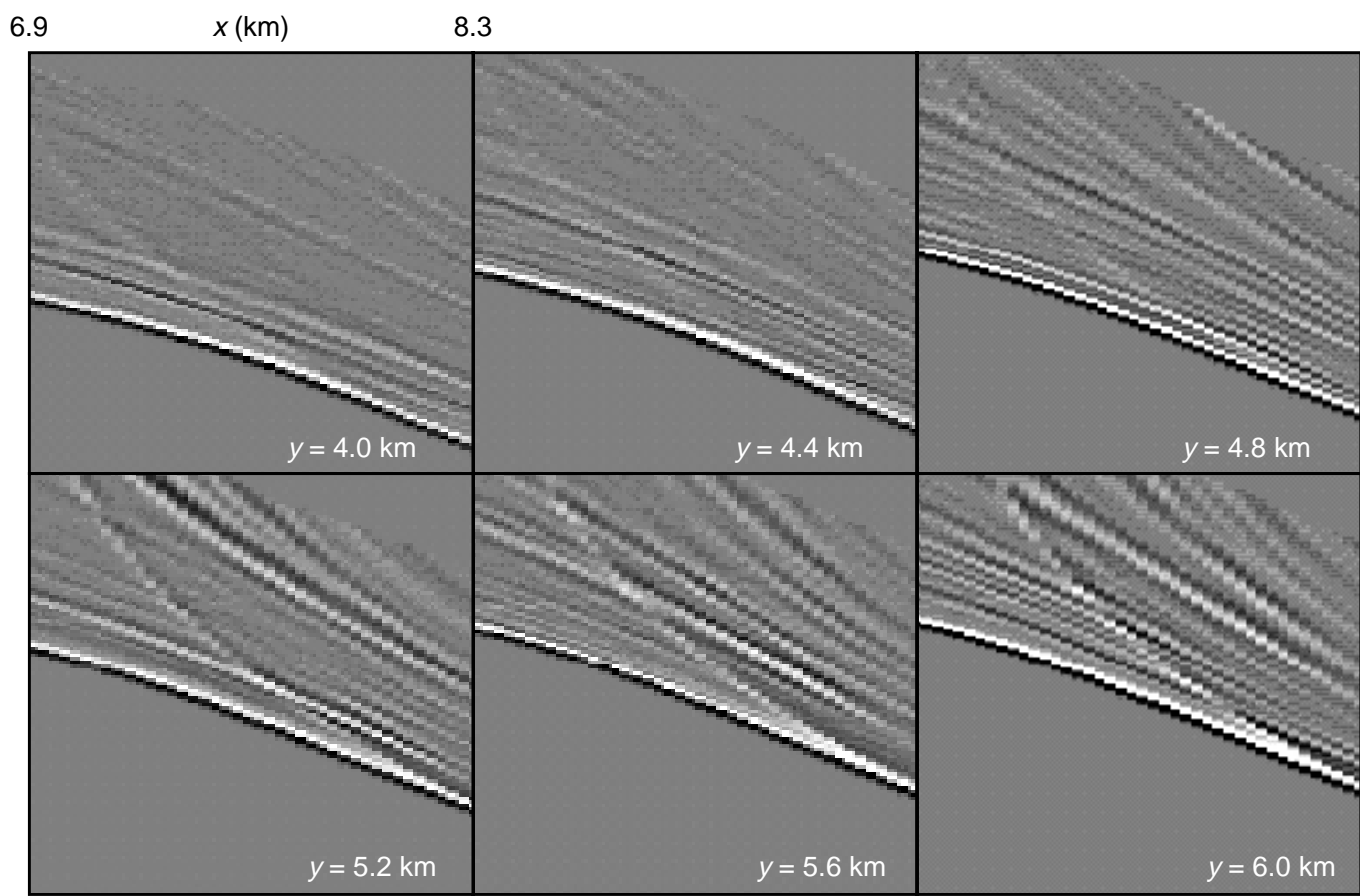

Figure 9

$y$ sections in the cube of offset class migrated images with $v=3000 \mathrm{~m} / \mathrm{s}$ and using the data associated with the marine survey shown in Figures $8 \mathrm{a}$ and $8 \mathrm{~b}$. The amplitude of the imaging artifacts is ten times lower than the one of the migrated event corresponding to the reflector of the model.

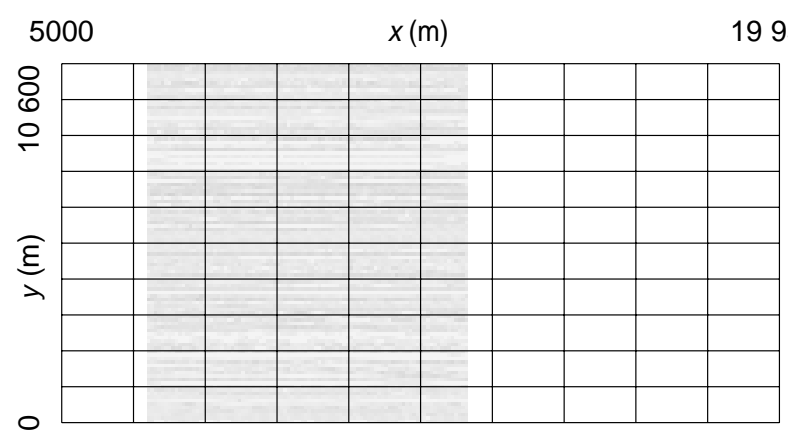

(a)

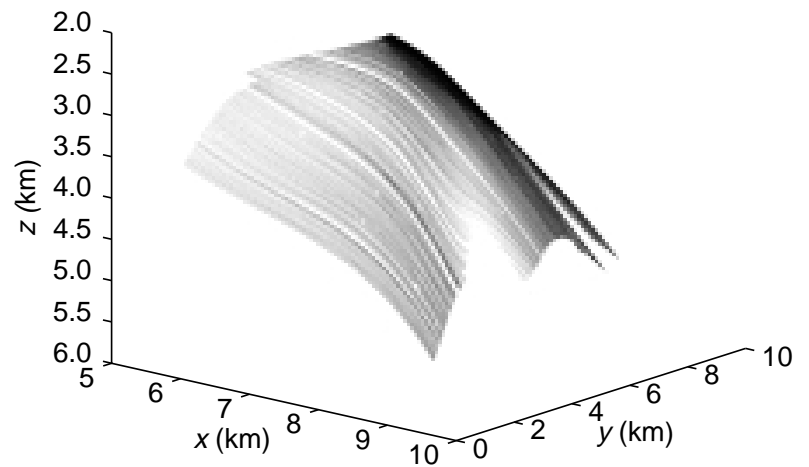

(b)

Figure 10

Survey from a marine acquisition in the North Sea.

(a) Map of locations of sources that have a receiver at a distance ranging within [2995 m; $3005 \mathrm{~m}$ ].

(b) $3 \mathrm{D}$ view of the impact points on the reflector. 


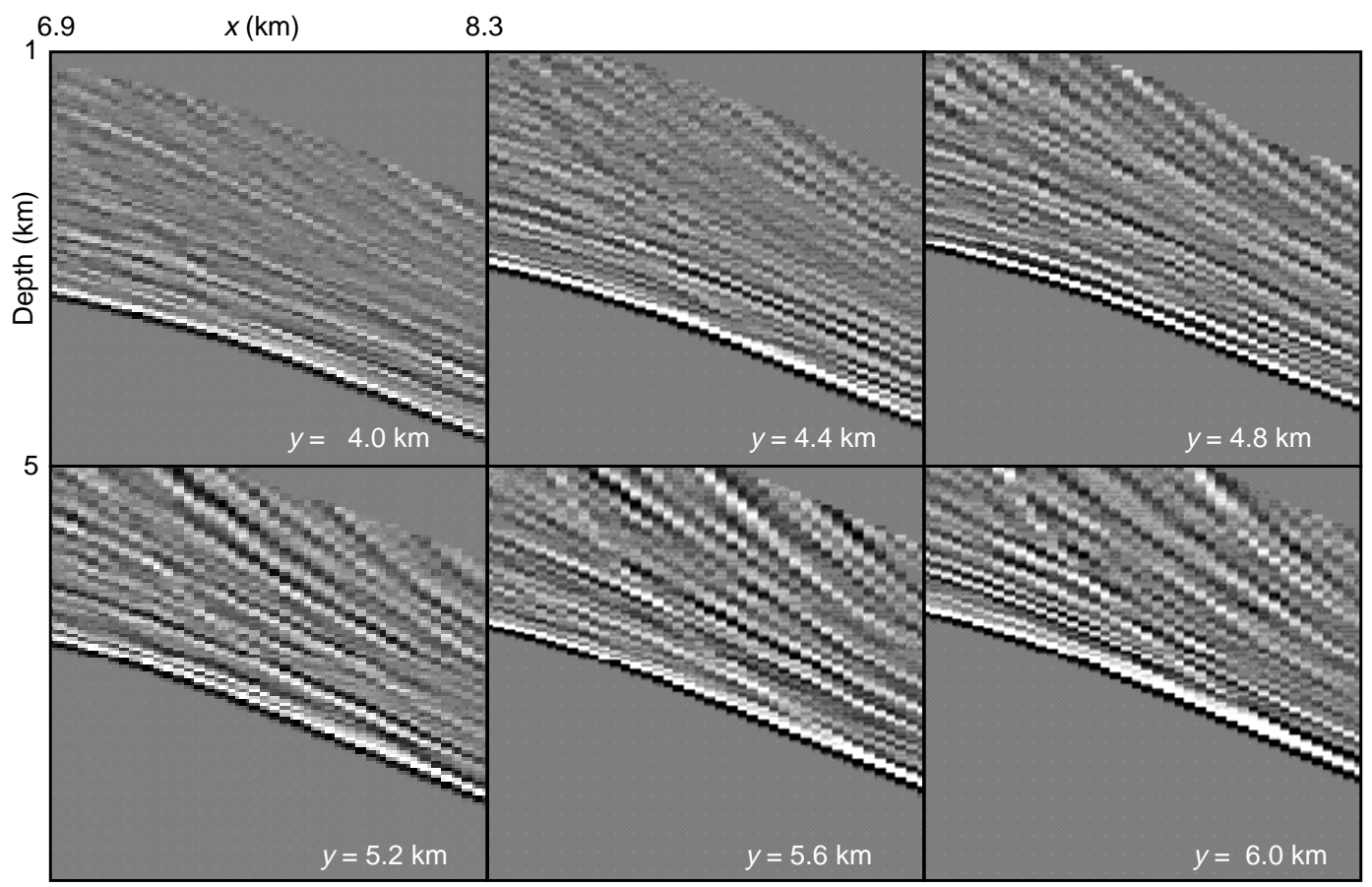

Figure 11

$y$ sections in the cube of offset class migrated images with $v=3000 \mathrm{~m} / \mathrm{s}$ and using the data associated with the marine survey shown in Figures $10 \mathrm{a}$ and $10 \mathrm{~b}$. The amplitude of the imaging artifacts is ten times lower than the one of the migrated event corresponding to the reflector of the model.

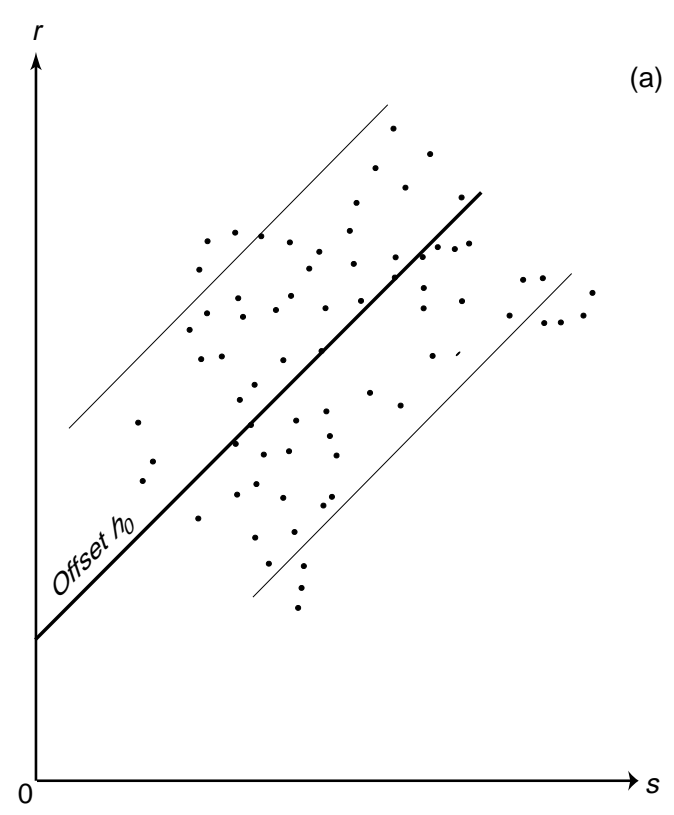

Figure 12a

Irregular seismic acquisition in 2D.

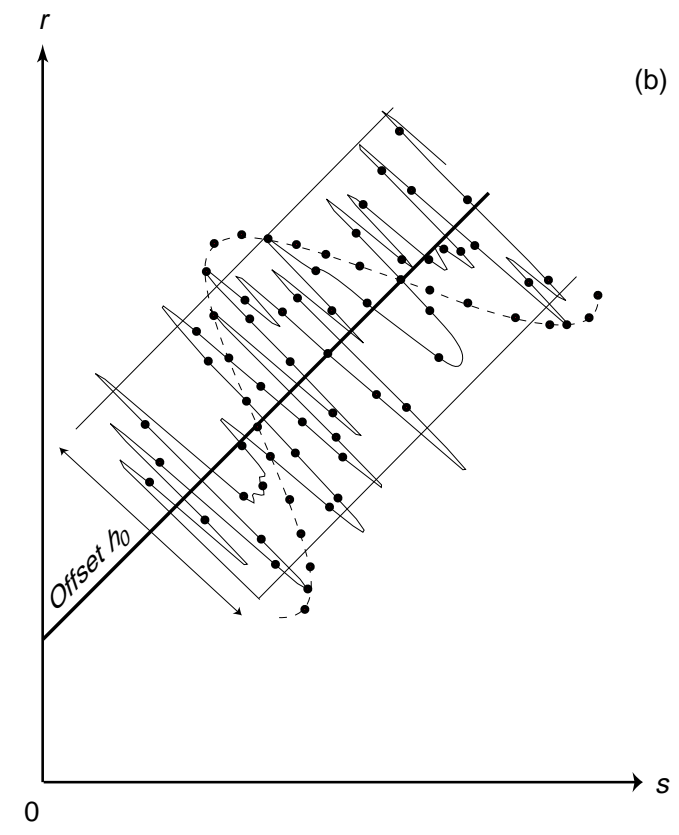

Figure $12 b$

Two possible choices of offset class function. The sourcereceiver pairs that constitute the offset class appear as samples of the graph of the selected offset class function. 


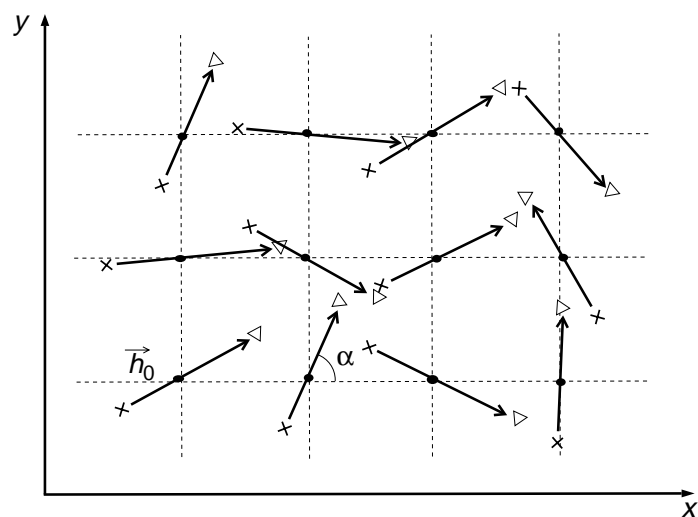

(a)

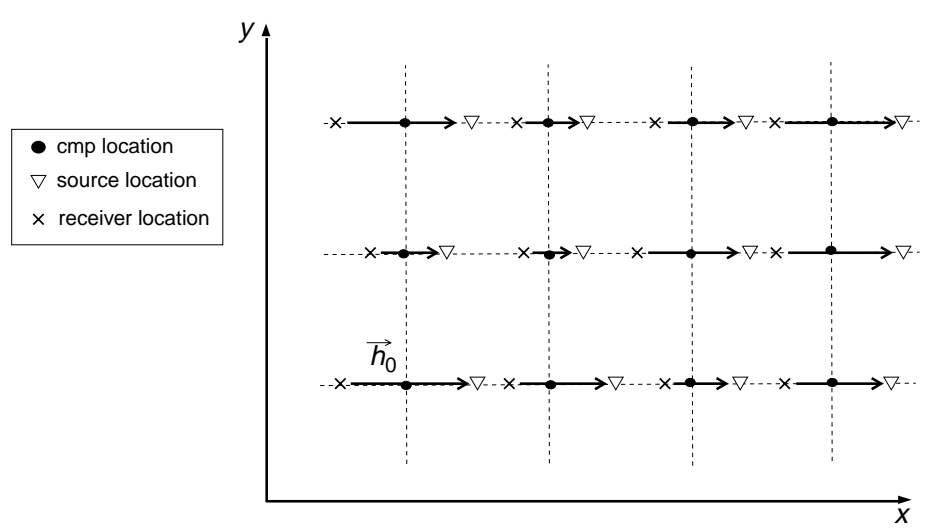

(b)

Figure 13

Examples of synthetic offset classes with a uniform distribution of midpoints.

(a) Mixing of azimuths. The norm of each offset is fixed to $\left\|\vec{h}_{0}\right\|$ and the azimuth $\alpha$ varies randomly.

(b) Mixing of norms. For a fixed azimuth (for instance here $\alpha=0$ ) the norm of each offset varies randomly around $\left\|\vec{h}_{0}\right\|$.

the nodes of a regular grid). To create a synthetic acquisition we consider a discrete function $w(k)$ that gives the sourcereceiver pair $\left(\vec{s}_{k}, \vec{r}_{k}\right)$ associated with node $k$ of the grid of midpoints: the values $w(k)$ define the samples of the considered offset class function. These values will be obtained from the realization of a random function, involving possible correlations.

\subsubsection{Fixed Source-Receiver Distance and Non-Correlated Random Mixing of Azimuths}

We consider here non-correlated variations in the azimuth: the values taken by the offset class function are those associated with the realization of a non-correlated random function with uniform density that defines the azimuth, the norm $\|\vec{h}\|$ being set to $3000 \mathrm{~m}$. We consider azimuth $\alpha$ uniformly distributed within $\left[-18^{\circ}, 18^{\circ}\right]$ and $\left[-90^{\circ}, 90^{\circ}\right]$, successively (for $\alpha \in\left[-18^{\circ}, 18^{\circ}\right]$ source locations are displayed in Figures 14a-14c). Figures 15 and 16 give the migrated cubes produced by migration by offset class. We observe that in both cases the geometry of the migrated event is the one of the reflector, which is not surprising since, for an exact migration velocity, all source-receiver pairs contribute to the construction of a single envelope (which is precisely the geometry of the reflector). Nevertheless the mixing of azimuths, involving fluctuations of offset in the data, introduces artifacts in the images; the larger the fluctuation in azimuths, the stronger is this contamination (we recall that these artifacts are not shown from $1 \mathrm{~km}$ above the reflector because of the restricted imaging domain). For the most critical azimuth range $\left[-90^{\circ}, 90^{\circ}\right.$, Figure 17 displays the section $y=5200 \mathrm{~m}$ of the migrated cube for different offset norms $\left(\left\|\vec{h}_{0}\right\|=100 \mathrm{~m}, 500 \mathrm{~m}, 1000 \mathrm{~m}, 2000 \mathrm{~m}, 2500 \mathrm{~m}\right.$ and $3000 \mathrm{~m}$ ). We observe that, for small source-receiver distances, artifacts are almost inexistent and that they increase with the offset norm. This is understandable as, in the limit, azimuth does not influence the zero offset data.

For an erroneous migration velocity $v=4000 \mathrm{~m} / \mathrm{s}$, offset norm $\left\|\vec{h}_{0}\right\|=3000 \mathrm{~m}$ and azimuth range $\left[-90^{\circ}, 90^{\circ}\right]$, artifacts due to the mixing of azimuths are added to the deformation of the reflector due to the velocity perturbation (Fig. 18). Nevertheless we observe that, surprisingly, the amplitude of artifacts is lower than those appearing with an exact migration velocity. Besides, it can be shown that artifacts increase with the offset norm also in the case of an erroneous migration velocity.

\subsubsection{Fixed Source-Receiver Distance and Correlated Random Mixing of Azimuths}

The values that define the azimuth at the different midpoints are given by the realization of a 2D Gaussian random function with exponential covariance. The covariance operator is defined by:

$$
C\left(\vec{q}_{1}, \vec{q}_{2}\right)=\sigma^{2} \exp \left(-\left\|\vec{q}_{1}-\vec{q}_{2}\right\| / \lambda\right)
$$

where:

- $\sigma^{2}$ is the variance;

- $\vec{q}_{1}$ and $\vec{q}_{2}$ define midpoint locations;

$-\lambda$ is the correlation length.

The variance governs the amplitude of the fluctuations of the random function and the correlation length governs the central frequency of these fluctuations.

For offset norm $\left\|\vec{h}_{0}\right\|=3000 \mathrm{~m}$, we present in Figures $19 \mathrm{a}-19 \mathrm{c}$ the source locations associated with a random 
function with an expectation of $-\pi / 2$, a standard deviation $\sigma$ of $\pi$ and a correlation length of $20 \mathrm{~km}$ (low-frequency fluctuations). The migrated cube obtained with these data (Fig. 20) presents artifacts which are not as strong as when the correlation length $\lambda=1 \mathrm{~km}$ (high-frequency fluctuations) (Figs. 21a-21c and 22). Hence as it was predicted by theory,

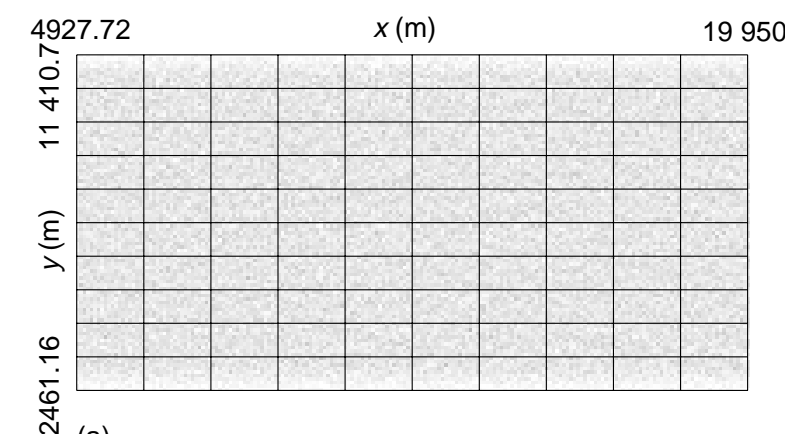

(a)

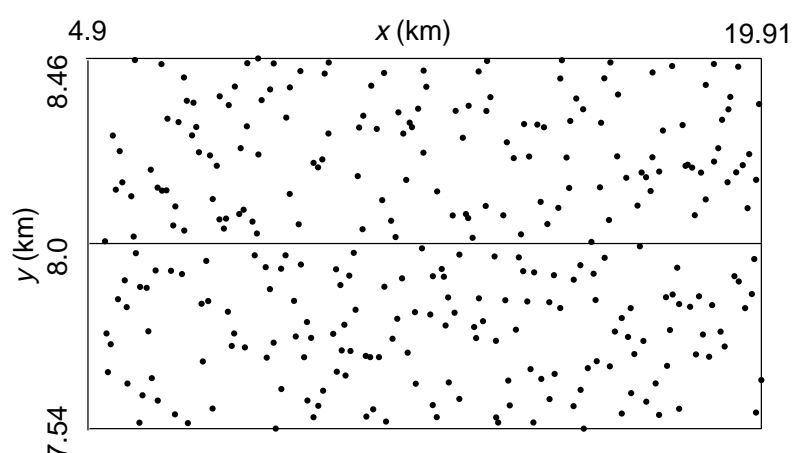

(b)

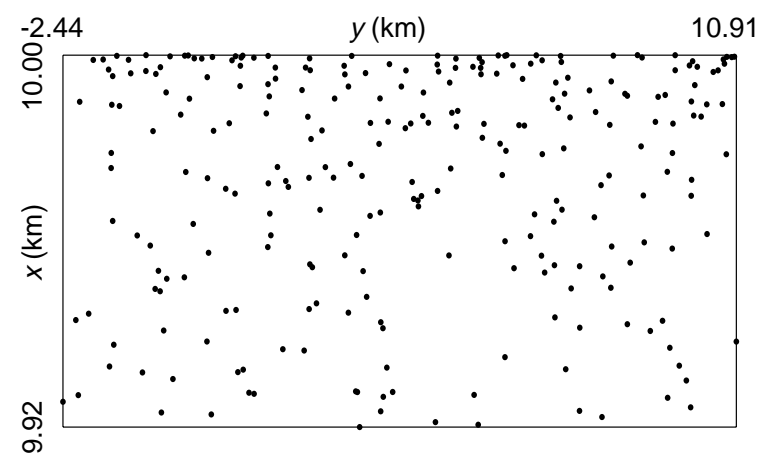

(C)

\section{Figure 14}

Source locations of a synthetic survey for $\left\|\vec{h}_{0}\right\|=3000 \mathrm{~m}$ and a non-correlated random mixing of azimuths ranging within $\alpha \in\left[-18^{\circ}, 18^{\circ}\right]$. The midpoint spacing is $50 \mathrm{~m}$ both in the $x$ and $y$ directions.

(a) Overview of source locations.
(b) Source locations for midpoints at $y=8000 \mathrm{~m}$.
(c) Source locations for midpoints at $x=10000 \mathrm{~m}$. high-frequency, i.e. non-smooth variations of the offset, gives rise to important artifacts: such variations must be avoided in the perspective of a high-quality imaging. For a fixed correlation length and azimuth range, we observe that, as for the non-correlated case, artifacts increase with offset norm (Figs. 23 and 24).

\subsubsection{Fixed Azimuth and Random Mixing of Norms}

In these experiments, for a fixed azimuth $\alpha=0$, the offset norm $\|\vec{h}\|$ varies randomly around a given offset norm $\left\|\vec{h}_{0}\right\|$. Figure 25 gives the section $y=5200 \mathrm{~m}$ of the migrated cube for an exact migration velocity, for $\left\|\vec{h}_{0}\right\|=3000 \mathrm{~m}$ and for source-receiver distances ranging randomly and without correlation within $[-5 \mathrm{~m}, 5 \mathrm{~m}],[-50 \mathrm{~m}, 50 \mathrm{~m}],[-500 \mathrm{~m}, 500$ $\mathrm{m}]$, successively. We observe that significant artifacts do not appear unless the range in source-receiver distance becomes unrealistic $([-500 \mathrm{~m}, 500 \mathrm{~m}])$. Even for that case, the amplitude of these artifacts is not as strong as for a low, noncorrelated mixing of azimuths $\alpha \in\left[-18^{\circ}, 18^{\circ}\right]$ (see Fig. 15 for comparison). Note also that the amplitude of these artifacts increases with average offset norm (Fig. 26).

Random mixing of norms involving spatial correlations yields similar results.

Thus, the mixing of norms within an offset class is not as critical as the mixing of azimuths regarding the quality of the imaging.

\subsection{Non-Uniform Distribution of Midpoints for a Fixed Offset}

We now study the effects on the migrated images of a nonuniform distribution of midpoints for a fixed offset.

For this we remove or duplicate lines in the regular survey in Figure 3a (source locations are respectively displayed in Figures 27a and 28a).

It is thus created local heterogeneities of density of midpoints, in the first case featured by holes and in the second one by overdensities.

Such a perturbation in the acquisition gives rise to strong artifacts and a drastic degradation in the quality of the imaging (Figs. $27 b$ and $28 b$, respectively).

With such an acquisition, we cannot expect the numerical formula (7) to approximate the continuous expression (8) for the migrated image: our oversimple diffraction stack, which is used in most 3D common offset migration industrial packages ${ }^{2}$ should be upgraded so as to compensate for non-uniformly sampled midpoints. Such an upgrade, which turns out to be no that simple, is the aim of some of our future studies,

(2) These industrial packages make use of filters (often called antialiasing filters) to remove these artifacts, which turns out to be effective but at the expense of the ability to image strongly dipping events. 


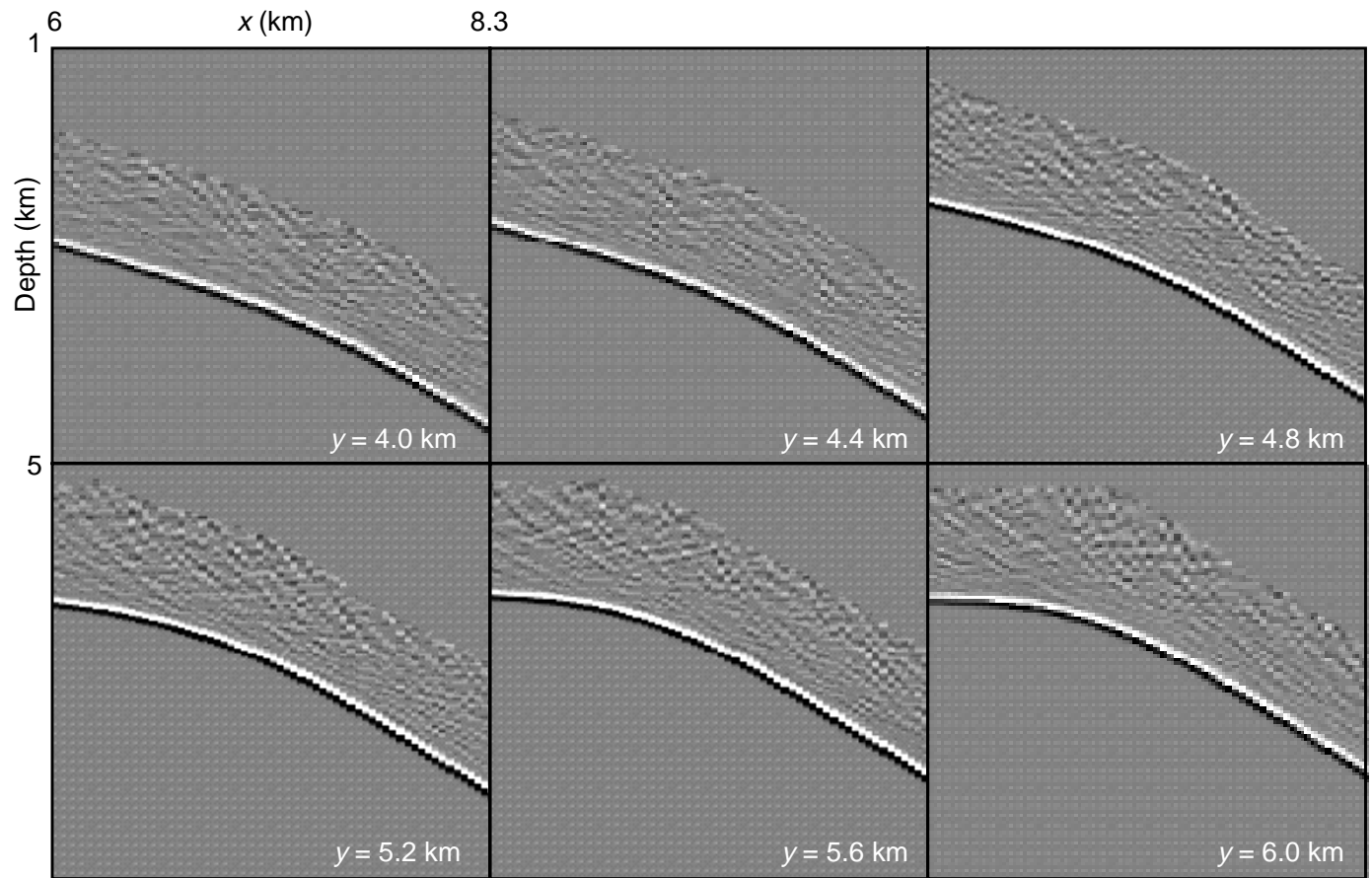

Figure 15

$y$ sections in the cube of offset class migrated images with $v=3000 \mathrm{~m} / \mathrm{s},\left\|\vec{h}_{0}\right\|=3000 \mathrm{~m}$ and non-correlated random azimuths uniformly distributed within $\left[-18^{\circ}, 18^{\circ}\right]$.

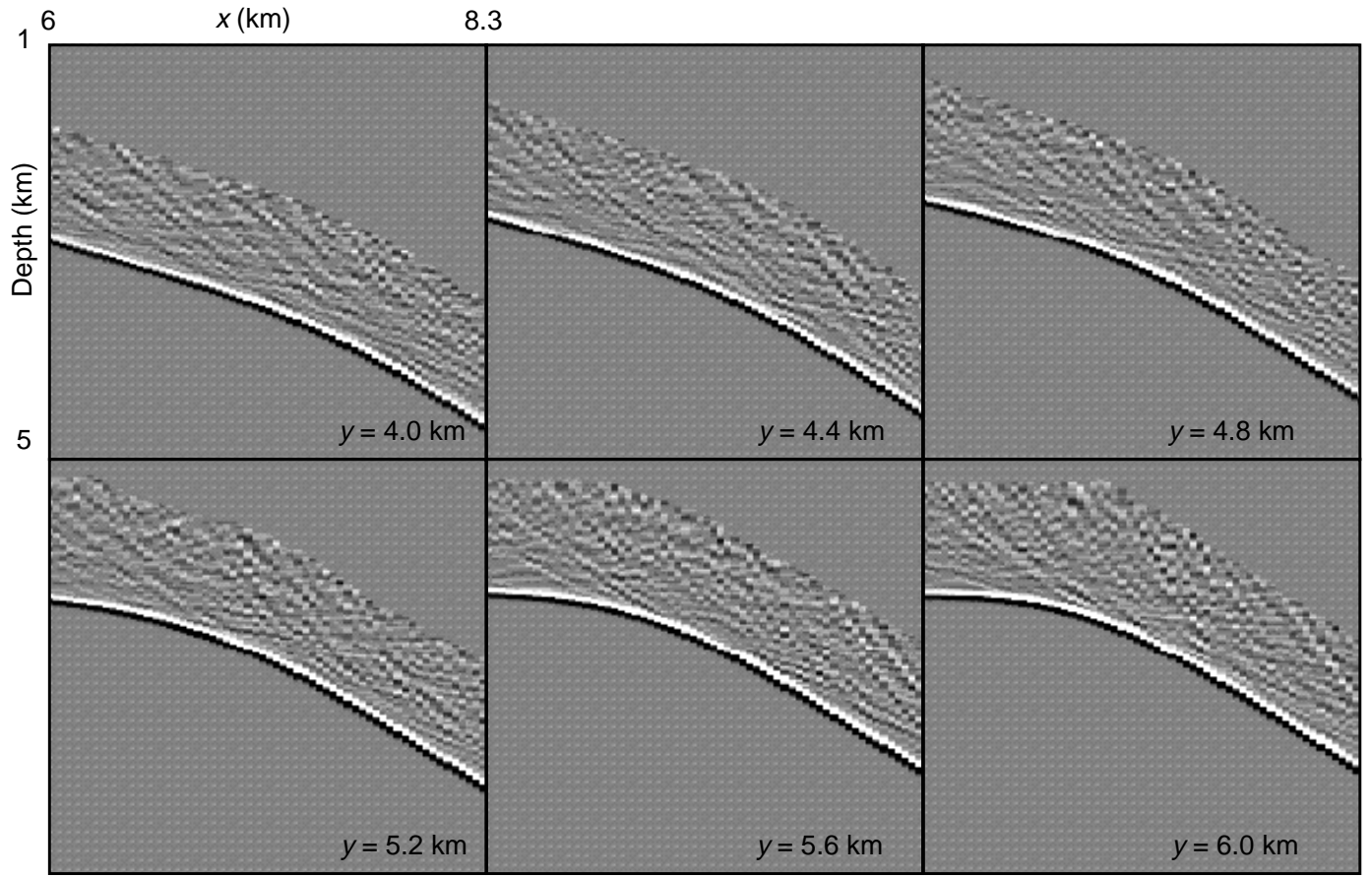

Figure 16

$y$ sections in the cube of offset class migrated images with $v=3000 \mathrm{~m} / \mathrm{s},\left\|\vec{h}_{0}\right\|=3000 \mathrm{~m}$ and non-correlated random azimuths uniformly distributed within $\left[-90^{\circ}, 90^{\circ}\right]$. 


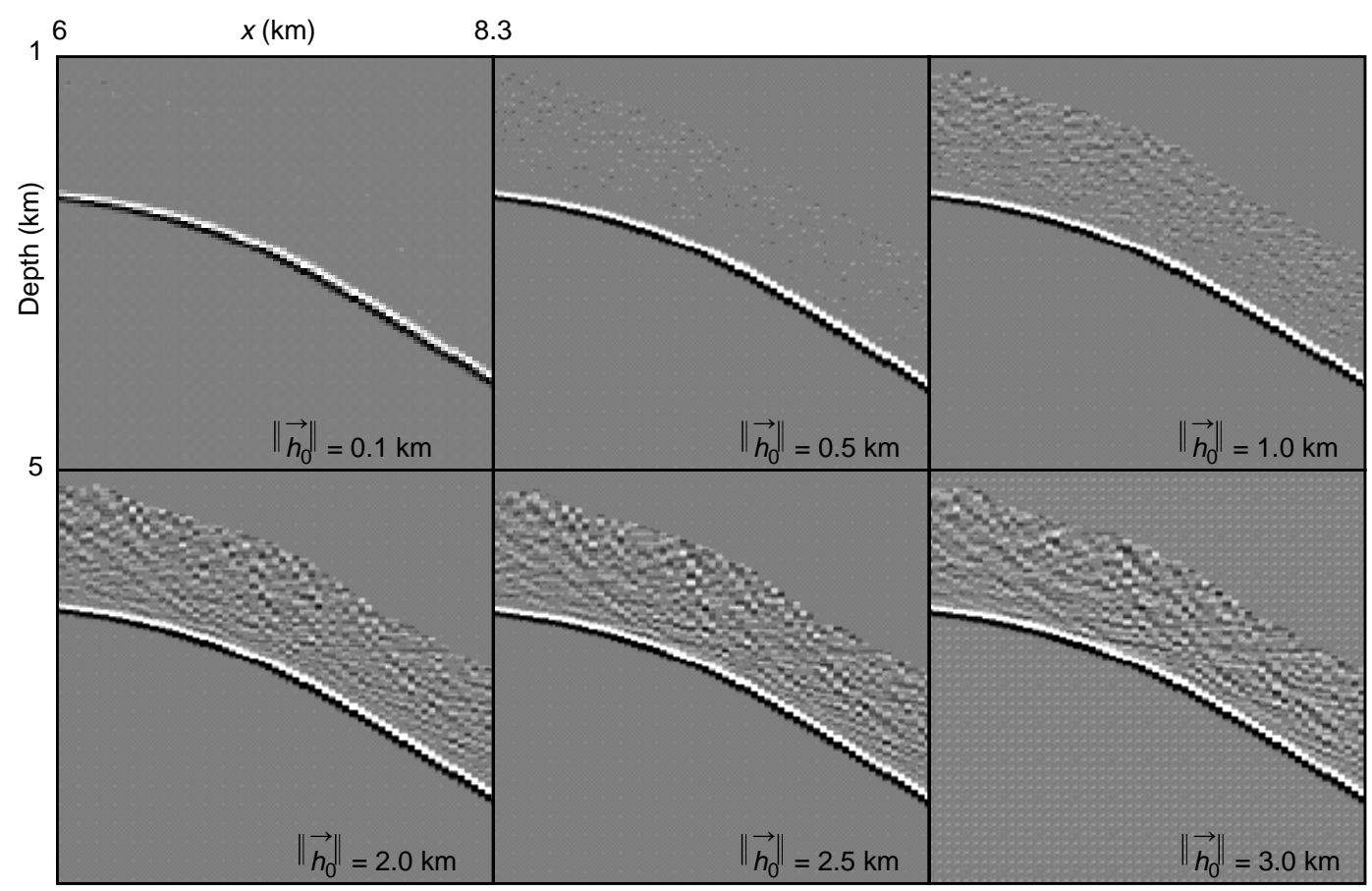

Figure 17

Sections $y=5.2 \mathrm{~km}$ in the cube of offset class migrated images with $v=3000 \mathrm{~m} / \mathrm{s}$, for different offset norms and non-correlated random azimuths uniformly distributed within $\left[-90^{\circ}, 90^{\circ}\right]$.

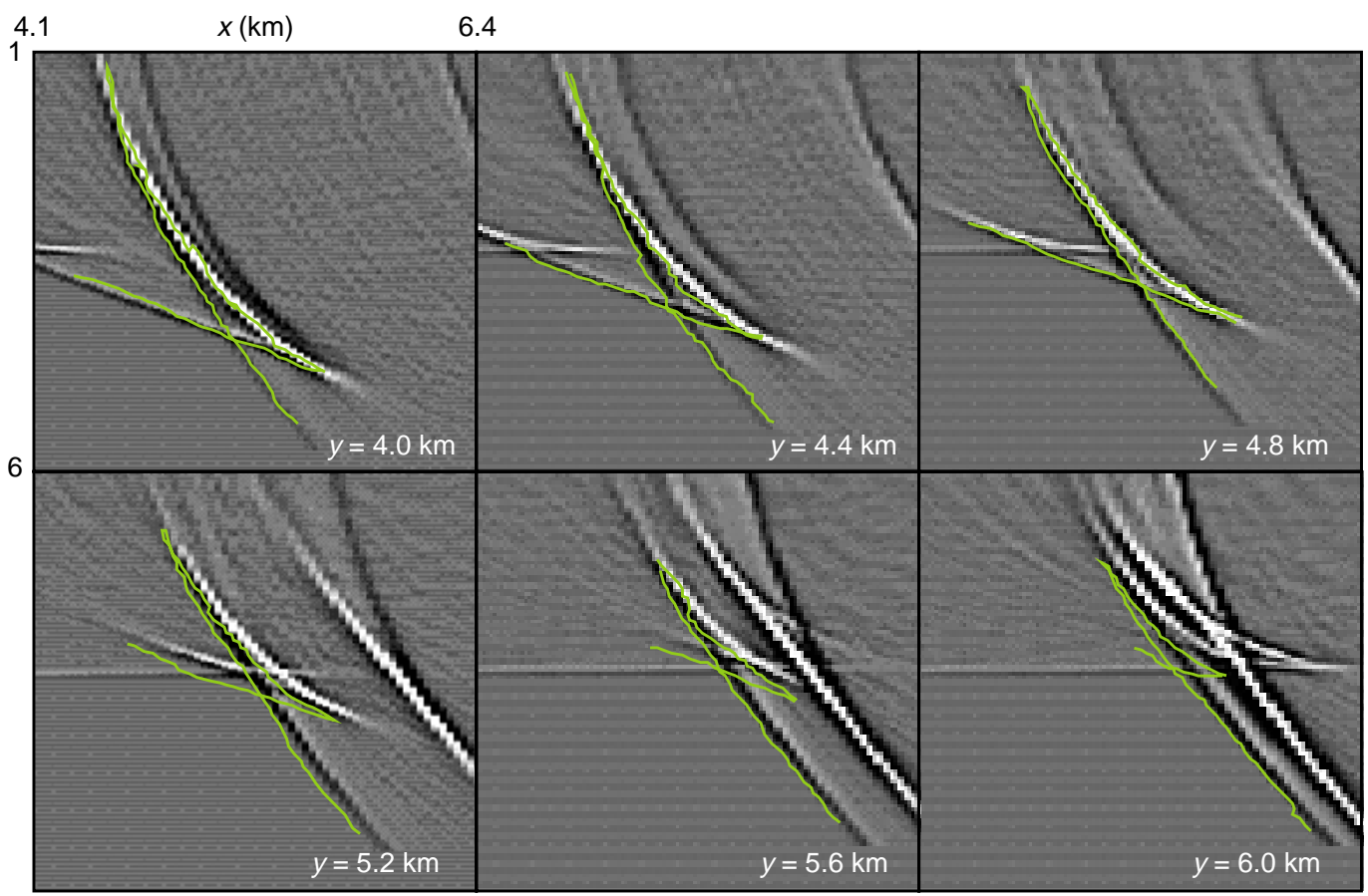

Figure 18

$y$ sections in the cube of offset class migrated images with a too high migration velocity $v=4000 \mathrm{~m} / \mathrm{s},\left\|\vec{h}_{0}\right\|=3000 \mathrm{~m}$ and non-correlated random azimuths uniformly distributed within $\left[-90^{\circ}, 90^{\circ}\right]$. The three cusp branches colored in green give the geometry of the migrated event. Other events are the so-called migration smiles due to data truncation. 


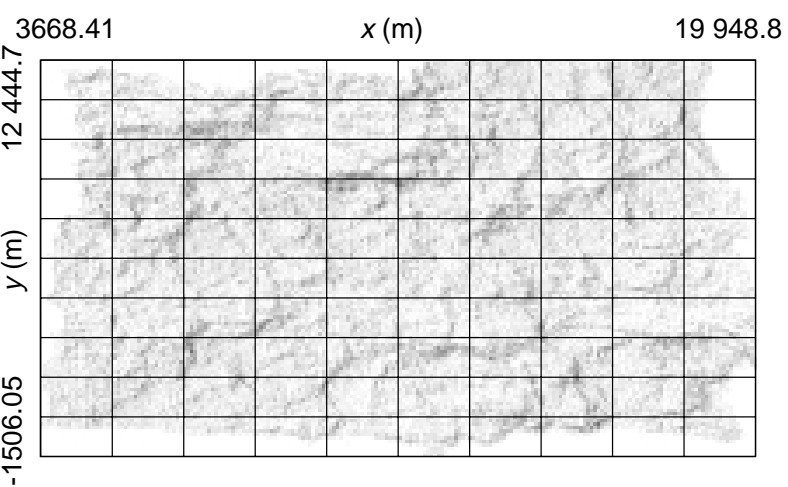

(a)

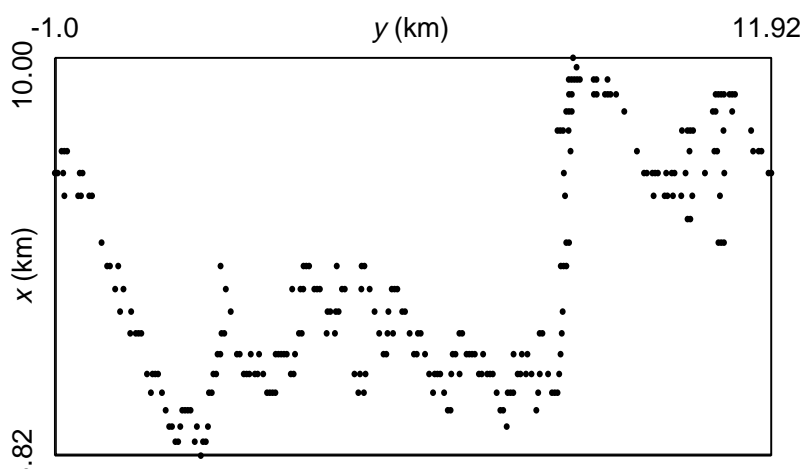

(c)

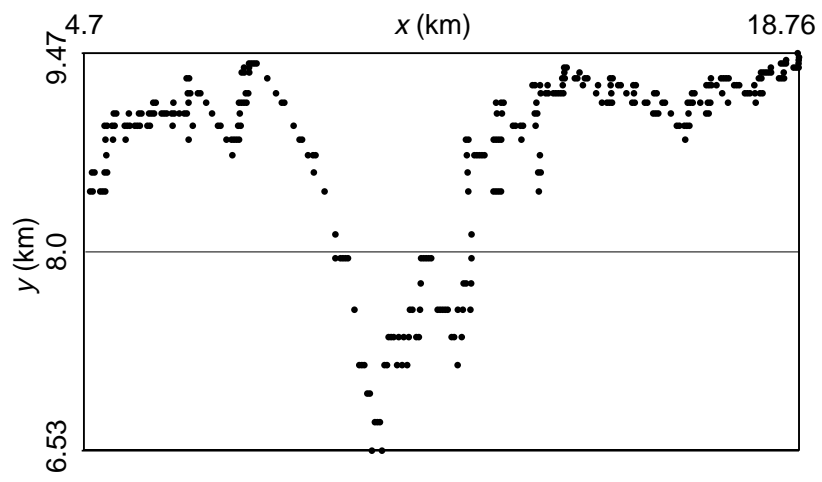

(b)

Source locations of a synthetic survey associated with a correlated random mixing of azimuths. The spacing between midpoints is $50 \mathrm{~m}$ both in the $x$ and $y$ directions and the source-receiver distance is fixed to $\left\|\vec{h}_{0}\right\|=3000 \mathrm{~m}$. The parameters that define the random function are: expectation $-\pi / 2$, exponential covariances with standard deviation $\sigma=\pi$ and correlation length $\lambda=20 \mathrm{~km}$.

(a) Overview of source locations.

(b) Source locations for midpoints at $y=8000 \mathrm{~m}$.

(c) Source locations for midpoints at $x=10000 \mathrm{~m}$.

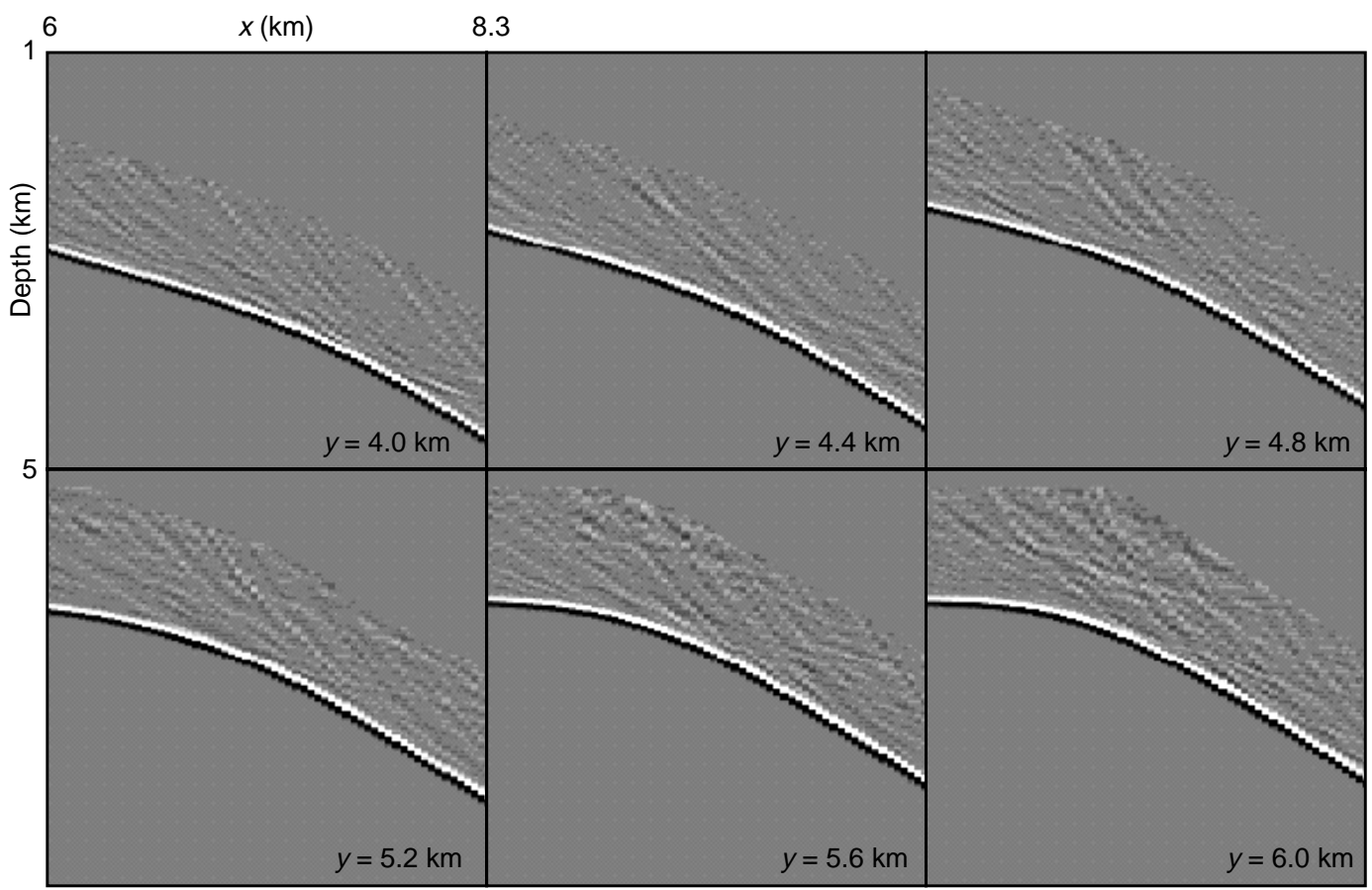

Figure 20

$y$ sections in the cube of offset class migrated images with $\left\|\vec{h}_{0}\right\|=3000 \mathrm{~m}, v=3000 \mathrm{~m} / \mathrm{s}$. The parameters associated with the random function that defines the azimuth are: expectation $-\pi / 2$, exponential covariances with standard deviation $\sigma=\pi$ and correlation length $\lambda=20 \mathrm{~km}$. 


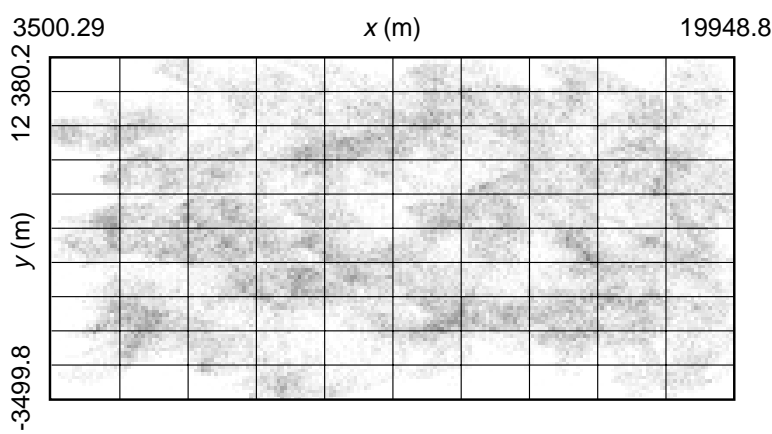

(a)

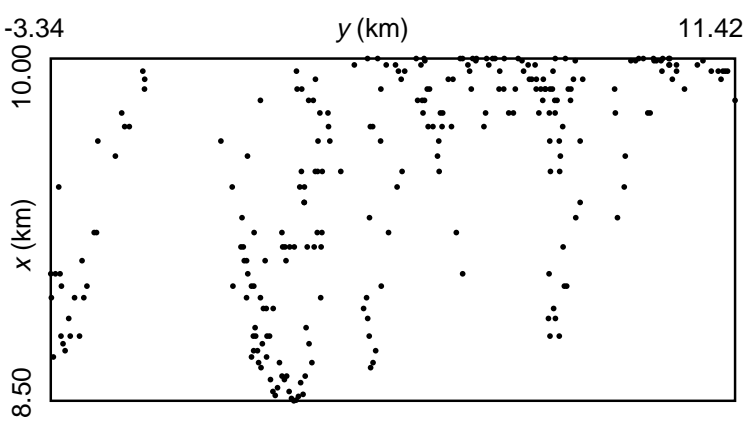

(c)

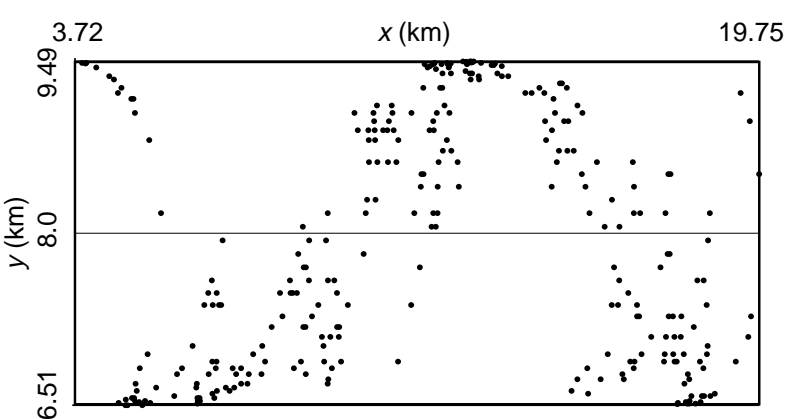

(b)

Source locations of a synthetic survey associated with a correlated random mixing of azimuths. The spacing between midpoints is $50 \mathrm{~m}$ both in the $x$ and $y$ directions and the source-receiver distance is fixed to $\left\|\vec{h}_{0}\right\|=3000 \mathrm{~m}$. The parameters that define the random function are: expectation $-\pi / 2$, exponential covariances with standard deviation $\sigma=\pi$ and correlation length $\lambda=1 \mathrm{~km}$.

(a) Overview of source locations.

(b) Source locations for midpoints at $y=8000 \mathrm{~m}$.

(c) Source locations for midpoints at $x=10000 \mathrm{~m}$.

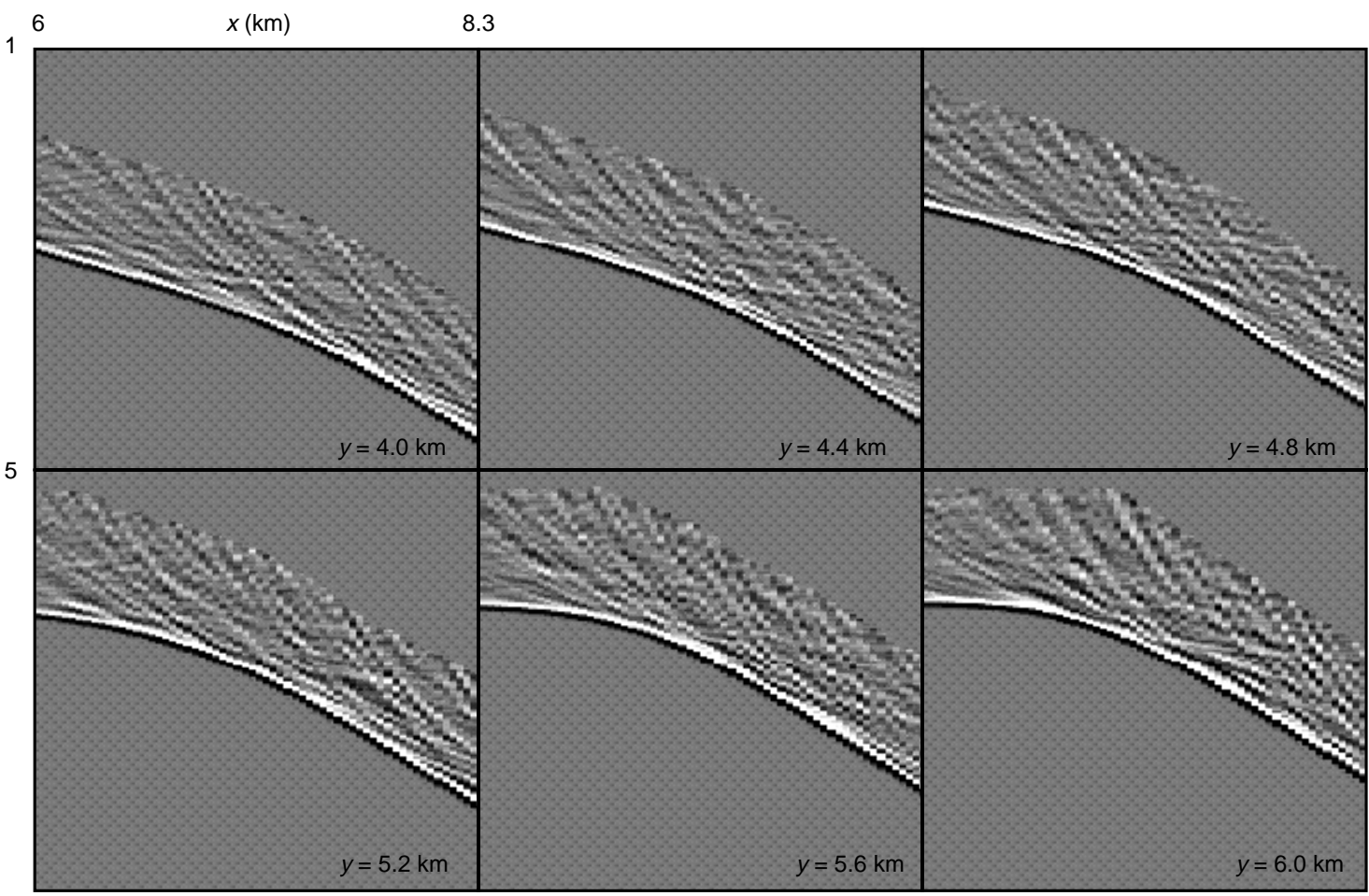

Figure 22

$y$ sections in the cube of offset class migrated images with $\left\|\vec{h}_{0}\right\|=3000 \mathrm{~m}, v=3000 \mathrm{~m} / \mathrm{s}$. The parameters associated with the random function that defines the azimuth are: expectation $-\pi / 2$, exponential covariances with standard deviation $\sigma=\pi$ and correlation length $\lambda=1 \mathrm{~km}$. 


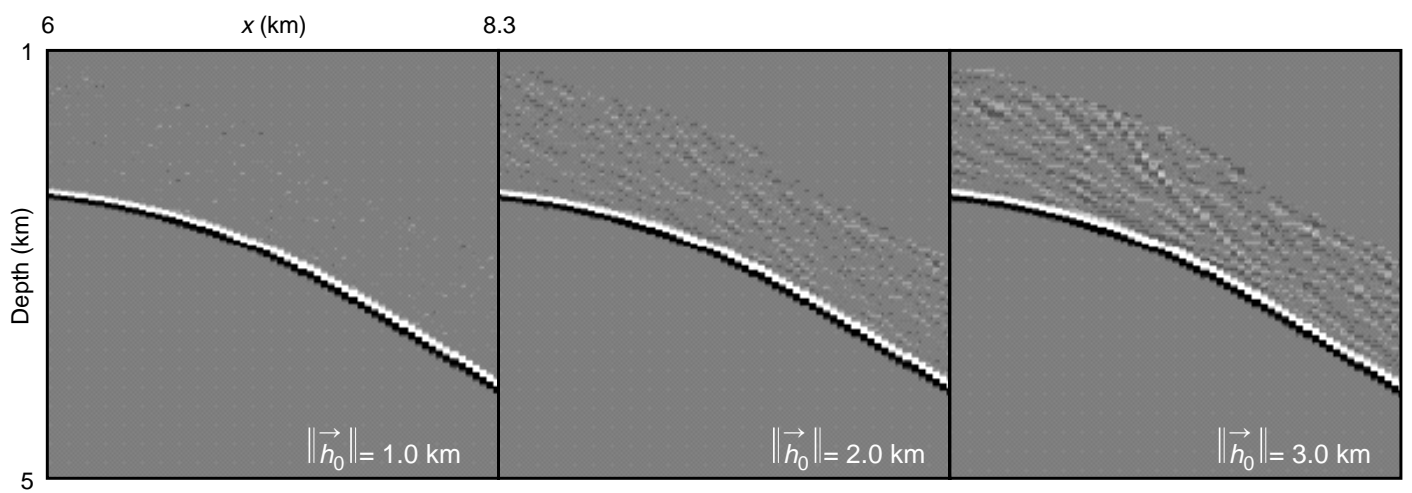

Figure 23

Sections $y=5.2 \mathrm{~km}$ in the cube of offset class migrated images with $v=3000 \mathrm{~m} / \mathrm{s}$, for different offset norms. The parameters associated with the random function that defines the azimuth are: expectation $-\pi / 2$, exponential covariances with standard deviation $\sigma=\pi$ and correlation length $\lambda=20 \mathrm{~km}$.

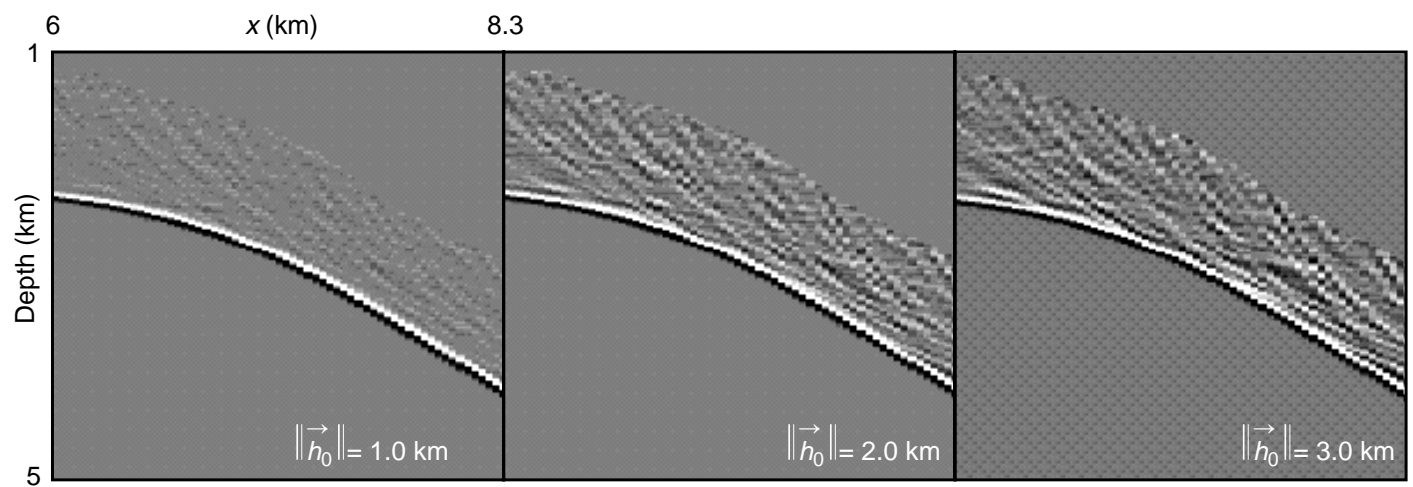

Figure 24

Sections $y=5.2 \mathrm{~km}$ in the cube of offset class migrated images with $v=3000 \mathrm{~m} / \mathrm{s}$, for different offset norms. The parameters associated with the random function that defines the azimuth are: expectation $-\pi / 2$, exponential covariances with standard deviation $\sigma=\pi$ and correlation length $\lambda=1 \mathrm{~km}$.

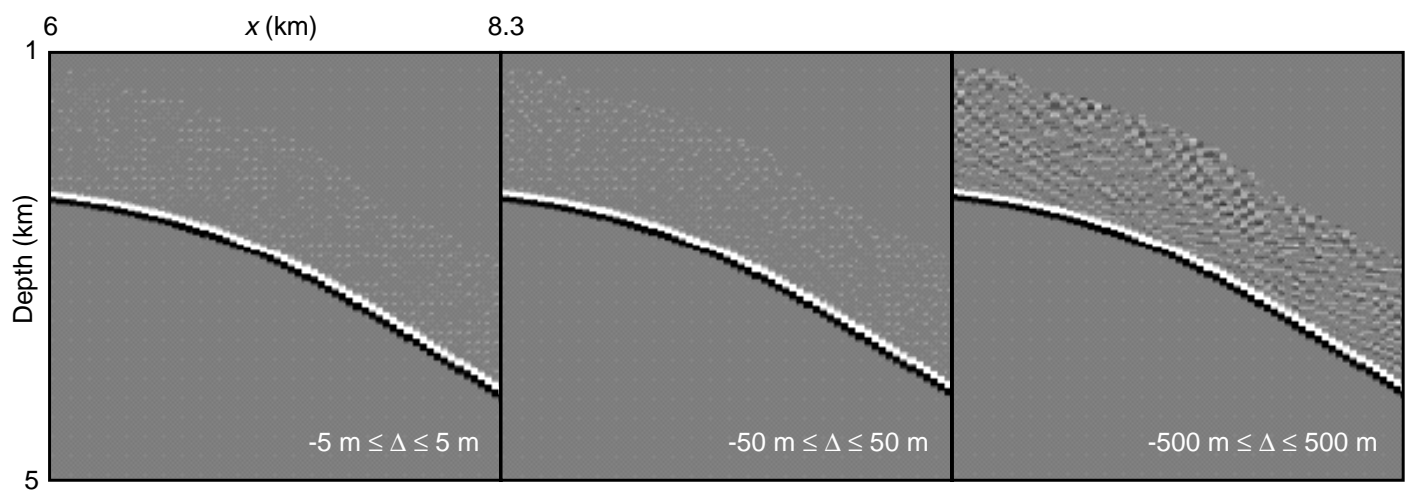

Figure 25

Sections $y=5.2 \mathrm{~km}$ in the cube of offset class migrated images with $v=3000 \mathrm{~m} / \mathrm{s}$, for an offset class with fixed azimuth $(\alpha=0)$ and a noncorrelated random fluctuation in source-receiver distance. The parameters that define these fluctuations are: average $3000 \mathrm{~m}$, range of fluctuations defined by parameter $\Delta$. 


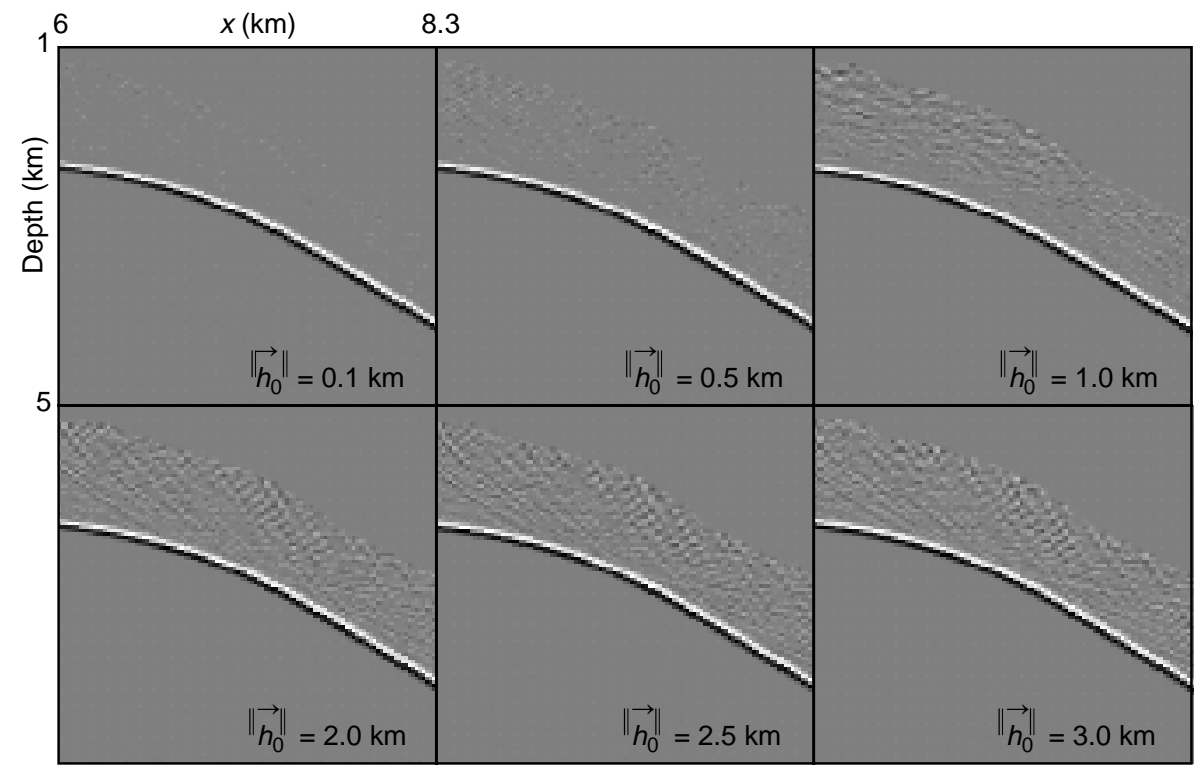

Figure 26

Sections $y=5.2 \mathrm{~km}$ in the cube of offset class migrated images with $v$ $=3000 \mathrm{~m} / \mathrm{s}$, for an offset class with fixed azimuth $(\alpha=0)$ and a noncorrelated random fluctuation in source-receiver distance. The parameters that define these fluctuations are: range of fluctuations $[-500 \mathrm{~m}$, $500 \mathrm{~m}]$, average defined by $\left\|\vec{h}_{0}\right\|$.

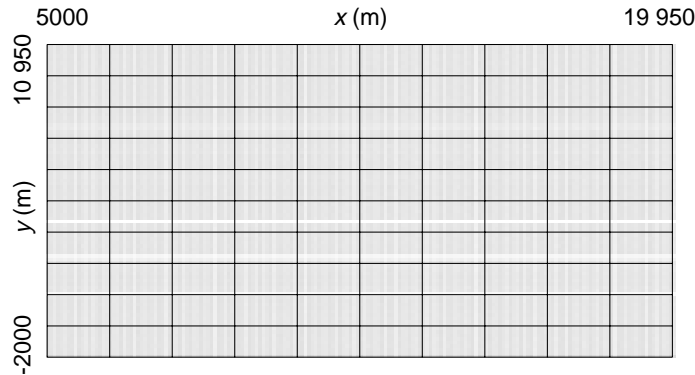

(a)

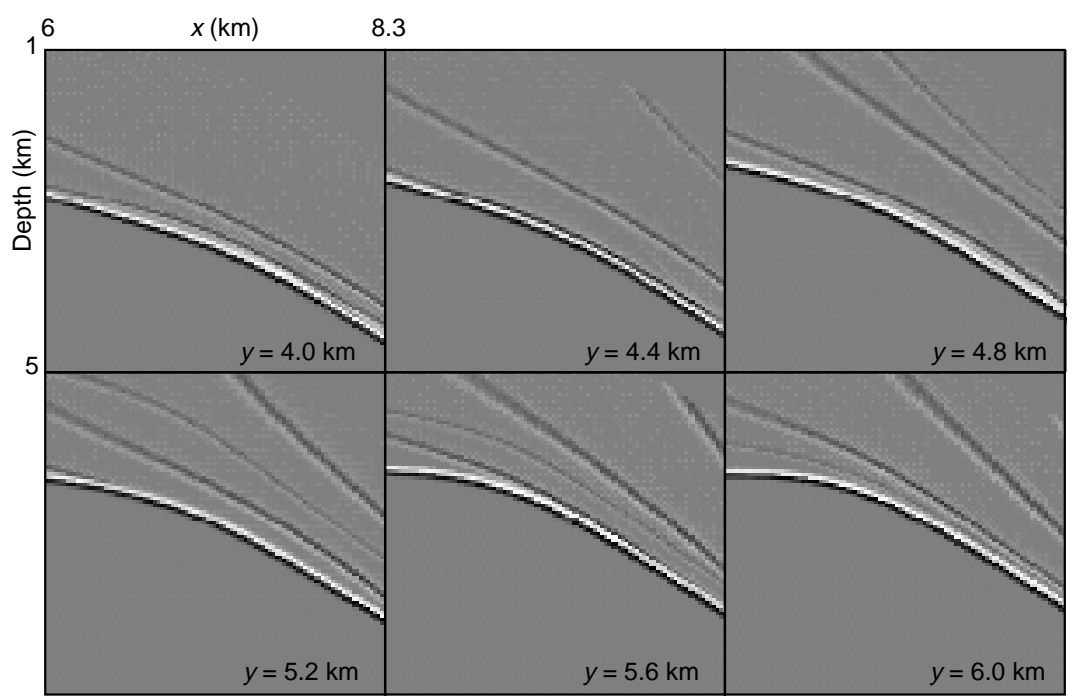

(b)

Figure 27

Local holes in the acquisition created by removing some in-lines in the regular survey shown in Figures 3a and $3 \mathrm{~b}$.

(a) Source locations.

(b) $y$ sections of the corresponding cube of offset class migrated images with $v=3000 \mathrm{~m} / \mathrm{s}$. 


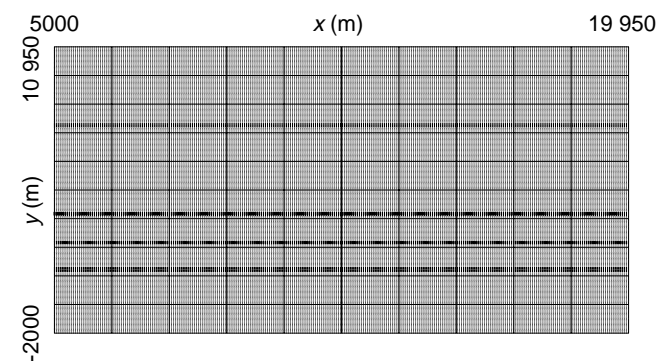

(a)

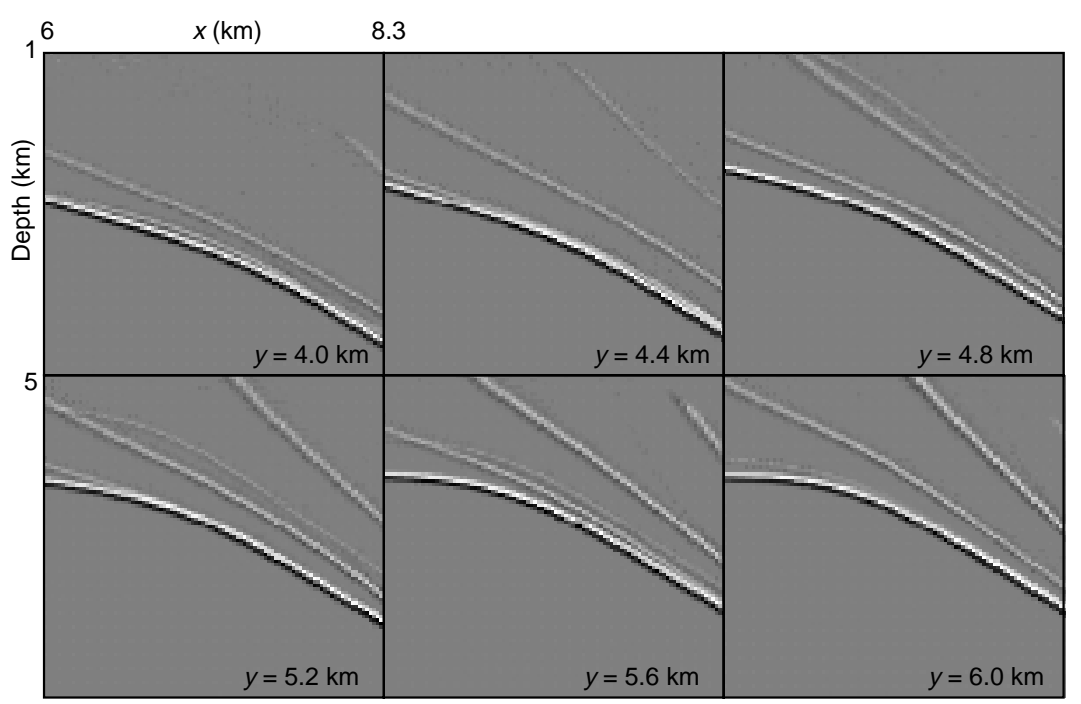

(b)

Figure 28

Local overdensities in the acquisition created by duplicating some in-lines in the regular survey shown in Figures $3 \mathrm{a}$ and $3 \mathrm{~b}$.

(a) Source locations.

(b) $y$ sections of the corresponding cube of offset class migrated images with $v=3000 \mathrm{~m} / \mathrm{s}$.

\section{CONCLUSION}

In 3D seismics, offset is a vector and irregularities in the acquisition make data associated with a given offset constituted of very few traces. In turn common offset migration does not make sense any longer: this notion is quite naturally extended to migration by offset class. Our goal was to analyze difficulties that can be met with such an imaging.

Offset class migration is a procedure that relies on a sound theoretical basis whenever the offset varies smoothly with the midpoint coordinates: seismic events give rise to migrated events whose supports are localized at the vicinity of the envelope of the isochrons, this whatever the migration velocity model (at least whenever it is smooth). However, this theory says almost nothing on how to constitute offset classes for a good imaging (we just know that smoothness is required): to what extent can we allow the offset to vary in norm, in azimuth? What is the influence, on the quality of the migrated images, of the distribution of midpoints involved in the offset class?

We have carried out an experimental study to answer those questions and, more generally, to understand how the offset classes have to be constituted. In a first part we have studied, for a uniform distribution of midpoints, the influence of the non-uniformity (in norm or in azimuth) of the different offsets involved. The conclusion is that we can mix substantially different offsets and this all the more than the offset varies slowly with the midpoint coordinate and that the mean offset is small. In a second part, we have studied, for a perfectly homogeneous distribution of offsets, the influence of a non-uniform distribution of midpoints. The conclusion is that the quality of the migrated image is very sensitive to such a non-uniformity: severe artifacts appear in case of missing traces (locally too small midpoint density), but also in case of overabundant data (locally too strong midpoint density), at least for the algorithm that we have used. 
From these observations different possibilities can be envisaged to constitute offset classes suited for a good imaging:

- use of interpolators to obtain a regular sampling in midpoints;

- use of a trace selection procedure yielding a distribution of midpoints as uniform as possible: elimination of traces giving rise to overabundant data, inclusion of traces with offsets far from the mean offset so as to fill holes in the coverage (we exploit here the robustness of the imaging regarding offset heterogeneities);

- use of a stacking formula more sophisticated than a mere diffraction stack, involving in particular weights that can account for a non-uniform distribution of midpoints.

Ongoing studies should provide us very soon with operational solutions.

\section{ACKNOWLEDGMENTS}

This research was carried out as part of the Kinematic Inversion Methods consortium project (KIM). The author hereby acknowledge the support provided by the sponsors of this project.

\section{REFERENCES}

Bleistein, N. (1984) Mathematical Methods for Wave Phenomena, Academic Press.

Bleistein, N. (1987) On the Imaging of Reflectors in the Earth. Geophysics, 52, 931-942.

Ehinger, A. and Lailly, P. (1995) Velocity Model Determination by the SMART Method, Part 1: Theory. 65th Ann. Internat. Mtg., Soc. Expl. Geophys., Expanded Abstracts, 739-742.

Géoltrain, S. and Léger, M. (1990) Kinematics of Migration, in PSI Annual Report, Institut français du pétrole, Rueil-Malmaison, France.

Jurado, F., Sinoquet, D. and Lailly, P. (1996) Jerry: a 3D Reflection Tomography Designed for Complex Structures, in KIM Annual Report, Institut français du pétrole, Pau, France.

Lailly, P. and Ehinger, A. (1991) Overview of the PSI Approach to Complex Structure Imaging, in PSI Annual Report, Institut français du pétrole, Rueil-Malmaison, France.

Schneider, W.A. (1978) Integral Formulation for Migration in Two and Three Dimensions. Geophysics, 43, 49-76..

Final manuscript received in August 2001 


\section{APPENDIX}

\section{KINEMATIC ANALYSIS OF OFFSET CLASS MIGRATION}

The goal of this Appendix is to give a theoretical basis to "offset class migration" and to provide us with insights into the kinematic properties of offset class migrated images. The theory below is nothing but a mere transposition of the theory of Bleistein (1987) to the specific case of offset class gathers and in the context of a simplified imaging formula (basically we do not pay attention to amplitudes). Our presentation follows the presentation that Géoltrain and Léger (1990) gave in the context of shot record migration.

Offset class migration, whose goal is to construct an image of a reflector from an aerial measurement of its reflected field and a given velocity field $v$, consists in migrating all traces that belong to a given offset class. Considering that the choice of an offset class is done, the set of source-receiver pairs is then described, in a continuous standpoint, by an offset class function $\tilde{h}$ that is assumed to remain in the vicinity of offset $\vec{h}$. Instead of the midpoint location $\vec{q}$, we have chosen to parameterize the study with the source location $\vec{s}$, so that the offset class function is now defined by:

$$
\hat{h}: \vec{s} \mapsto \hat{h}(\vec{s})=\vec{r}-\vec{s}
$$

The offsets now depend on the source location $\vec{s}$.

Note that the assumed existence of an offset class function implies that, this function being defined, there is a unique receiver $\vec{r}$ associated with any source $\vec{s}$. We will also assume that this function is continuously differentiable.

Note that, thanks to the assumptions above, it is easy to construct the function $\tilde{h}(\vec{q})$ once $\hat{h}(\vec{s})$ is known:

$$
\tilde{h}(\vec{q})=\tilde{h}\left(\vec{s}+\frac{\hat{h}(\vec{s})}{2}\right)
$$

The definition (8) of the offset class migrated image can be expressed in terms of the new offset class function $\hat{h}$ :

$$
m_{\hat{h}}(M)=\int_{S} m_{e l}^{v}\left(M ;(\vec{s}, \vec{s}+\hat{h}(\vec{s}))\left|\frac{\mathrm{d} \vec{q}}{\mathrm{~d} \vec{s}}\right| d^{2} \vec{s}\right.
$$

where $S$ is the set of sources that belong to the considered offset class $\hat{h}$ and $|\mathrm{d} \vec{q} / \mathrm{d} \vec{s}|$ is the Jacobian of the map:

$$
\vec{s} \mapsto \vec{q}=\vec{s}+\frac{\hat{h}(\vec{s})}{2}
$$

More precisely, with a kinematic version of "Prestack Kirchhoff Depth Migration", we can rewrite the definition of a migrated image:

$$
\begin{aligned}
& m_{\hat{h}}(M)= \\
& \int \mathrm{d} \omega i \omega \int_{S}\left|\frac{\mathrm{d} \vec{q}}{\mathrm{~d} \vec{s}}\right| \mathrm{d}(\vec{s}, \vec{s}+\hat{h}(\vec{s}), \omega) e^{-i \omega t_{m}(M ; \vec{s}, \vec{s}+\hat{h}(\vec{s}))} \mathrm{d}^{2} \vec{s}
\end{aligned}
$$

where:

$$
\begin{aligned}
& \mathrm{d}(\vec{s}, \vec{s}+\hat{h}(\vec{s}), \omega)= \\
& f(\omega) D_{e}(\vec{s}, \vec{s}+\hat{h}(\vec{s})) e^{i \omega t_{e}(\vec{s}, \vec{s}+\hat{h}(\vec{s}))}+O(1 / i \omega)
\end{aligned}
$$

is the asymptotic representation in the Fourier domain of the record associated with the source-receiver pair parameterized by $(\vec{s}, \vec{s}+\hat{h}(\vec{s}))$, with:

- $D_{e}(\vec{s}, \vec{s}+\hat{h}(\vec{s}))$, the specular scattering amplitude;

- $\omega$, the angular frequency;

- and $f(\omega)$, the source spectrum.

We recall that $t_{m}(M ; \vec{s}, \vec{s}+\hat{h}(\vec{s}))$ and $t_{e}(\vec{s}, \vec{s}+\hat{h}(\vec{s}))$ are the total traveltime from source and receiver to $M$ and the arrival time of the considered event, respectively.

Then:

$$
\begin{aligned}
m_{\hat{h}}(M)= & \int \mathrm{d} \omega i \omega f(\omega) \int_{S} \mathrm{~d}^{2} \vec{s} \\
& {\left[N(\vec{s}) e^{i \omega\left[t_{e}(\vec{s}, \vec{s}+\hat{h}(\vec{s}))-t_{m}(M ; \vec{s}, \vec{s}+\hat{h}(\vec{s}))\right]}+O(1 / i \omega)\right] }
\end{aligned}
$$

where:

$$
N(\vec{s})=\left|\frac{\mathrm{d} \vec{q}}{\mathrm{~d} \vec{s}}\right| D_{e}(\vec{s}, \vec{s}+\hat{h}(\vec{s}))
$$

We introduce the phase:

$$
\Phi(M ; \vec{s})=t_{e}(\vec{s}, \vec{s}+\hat{h}(\vec{s}))-t_{m}(M ; \vec{s}, \vec{s}+\hat{h}(\vec{s}))
$$

We assume that $\Phi$ has a stationary point $\vec{s}^{m}$ of order one (higher orders are ignored since they exist only for isolated values of $M$ ), namely:

$$
\frac{\partial \Phi}{\partial s_{i}}\left(M ; \vec{s}^{m}\right)=0, \quad \frac{\partial^{2} \Phi}{\partial s_{i}^{2}}\left(M ; \vec{s}^{m}\right) \neq 0, \quad i=x, y
$$

Considering that $f(\omega)$ is a high-pass filter, the multidimensional stationary phase method (see for instance Bleistein, 1984) provides an evaluation of the $\vec{s}$-integral in the high-frequency limit, namely:

$m_{\hat{h}}(M)=H\left(M ; \vec{s}^{m}\right) \int \mathrm{d} \omega f(\omega)\left[e^{i \omega \Phi\left(M ; \vec{s}^{m}\right)}+O(1 / i \omega)\right](11)$

where:

$$
\left|H\left(M ; \vec{s}^{m}\right)\right|=\sqrt{\frac{2 \pi}{\left|\frac{\partial^{2}\left(t_{e}-t_{m}\right)}{\partial s_{x} \partial s_{y}}\left(M ; \vec{s}^{m}, \vec{s}^{m}+\hat{h}\left(\vec{s}^{m}\right)\right)\right|}}\left|N\left(\vec{s}^{m}\right)\right|
$$

is an amplitude term which is independent of frequency.

Consequently, the migrated image is a bandlimited singular function with support on points $M$ that satisfy the zero-phase condition: 


$$
\Phi\left(M ; \vec{s}^{m}\right)=0
$$

Thus, the geometry of the migrated event is given by the set of points $M$ that satisfy both zero-phase and stationary phase conditions:

$$
\left\{\begin{array}{l}
t_{m}(M ; \vec{s}, \vec{s}+\hat{h}(\vec{s}))-t_{e}(\vec{s}, \vec{s}+\hat{h}(\vec{s}))=0 \\
\frac{\partial}{\partial s_{i}}\left[t_{m}(M ; \vec{s}, \vec{s}+\hat{h}(\vec{s}))-t_{e}(\vec{s}, \vec{s}+\hat{h}(\vec{s}))\right]=0 \quad i=x, y
\end{array}\right.
$$

It is the envelope of the family of isochrons generated by the different shots.

\section{Stationary Phase Condition and Rays}

The goal of this section is to characterize the set of points $M$ that satisfy, for a given $\vec{s}$, the stationary phase condition:

$$
\frac{\partial t_{m}}{\partial s_{i}}(M ; \vec{s}, \vec{s}+\hat{h}(\vec{s}))=\frac{\partial t_{e}}{\partial s_{i}}(\vec{s}, \vec{s}+\hat{h}(\vec{s})) \quad i=x, y
$$

The right-hand side in Equation (13) is interpreted as the slope at $\vec{s}$, in the $i$ direction, of the event in the offset class gather: this quantity is known (it is directly read in the data). To evaluate:

$$
\frac{\partial t_{m}}{\partial s_{i}}(M ; \vec{s}, \vec{s}+\hat{h}(\vec{s}))
$$

we have to evaluate the first-order perturbation in the total traveltime $t_{m}$ that results from a perturbation in the source location.

A perturbation $\mathrm{d} \vec{s}$ in the source location gives rise to a perturbation $\mathrm{d} \vec{r}$ in the receiver location so as to follow the considered offset class:

$$
\mathrm{d} r_{i}=\mathrm{d} s_{i}+\frac{\partial \hat{h}_{i}}{\partial s_{j}}(\vec{s}) \mathrm{d} s_{j} \quad i, j=x, y
$$

and therefore the total traveltime:

$$
t_{m}(M ; \vec{s}, \vec{s}+\hat{h}(\vec{s}))=t(M ; \vec{s})+t(M ; \vec{r}=\vec{s}+\hat{h}(\vec{s}))
$$

$(t(M ; \vec{s})$ and $t(M ; \vec{r})$ are the traveltimes between point $M$ and source $\vec{s}$ and between point $M$ and receiver $\vec{r}$, respectively) is perturbed of an amount $\mathrm{d} t_{m}$ :

$$
\mathrm{d} t_{m}=\frac{\partial t}{\partial s_{i}} \mathrm{~d} s_{i}+\frac{\partial t}{\partial r_{i}} \mathrm{~d} r_{i}=-p_{i}^{\vec{s}} \mathrm{~d} s_{i}+p_{i}^{\vec{r}} \mathrm{~d} r_{i} \quad i=x, y
$$

where $p_{i}^{\vec{s}}$ and $p_{i}^{\vec{r}}$ are the $i$ components of the ray parameter of the ray starting from source at $\vec{s}$ and reaching $M$ and of the ray starting from $M$ and reaching receiver at $\vec{r}$, respectively.
Note that we use Einstein's summation convention on repeated indices.

Thus the perturbation $\mathrm{d} t_{m}$ is linked to $\mathrm{d} \vec{s}$ by:

and $\partial t_{m} / \partial s_{i}$ is given by:

$$
\mathrm{d} t_{m}=-p_{i}^{\vec{s}} \mathrm{~d} s_{i}+p_{i}^{\vec{r}}\left[\mathrm{~d} s_{i}+\frac{\partial \hat{h}_{i}}{\partial s_{j}}(\vec{s}) \mathrm{d} s_{j}\right]
$$

$\frac{\partial t_{m}}{\partial s_{i}}(M ; \vec{s}, \vec{s}+\hat{h}(\vec{s}))=-p_{i}^{\vec{s}}+p_{i}^{\vec{r}}+\frac{\partial \hat{h}_{j}}{\partial s_{i}}(\vec{s}) p_{j}^{\vec{r}} \quad i, j=x, y$

Therefore the stationary phase condition (13) defines a set of points $M$ located both on the ray that starts from the source at $\vec{s}$ with the direction given by $p_{i}^{\vec{s}}$ ( $p_{i}^{\vec{s}}$ is now a parameter) and on the ray that starts from $M$ and reaches receiver at $\vec{s}+\hat{h}(\vec{s})$ with a direction given by $p_{i}^{\vec{r}}$, solution of the system:

$$
p_{i}^{\vec{r}}+\frac{\partial \hat{h}_{j}}{\partial s_{i}}(\vec{s}) p_{j}^{\vec{r}}=p_{i}^{\vec{s}}+\frac{\partial t_{e}}{\partial s_{i}}(\vec{s}, \vec{s}+\hat{h}(\vec{s})) \quad i, j=x, y
$$

The set of such points $M$ appears as one-dimensional (considering the coordinates $(x, y, z)$ of $M$ we can deduce $\vec{p}^{\vec{s}}$ and $\vec{p}^{\vec{r}}$ and System (14) gives two relations that link the three coordinates). The intersection of this one-dimensional set with the isochron will, of course, give the point of the isochron that contributes in the migrated event.

\section{Dip of a Migrated Event}

System (12) also gives indications on the dip of the migrated event at point $M$.

At $M$ the dip of the migrated event (plane that is tangent to the envelope) is the plane that is tangent to the isochron. This plane is, as it is well known (it is the generalization of the result that states that the normal to an ellipse at a point $M$ is the bisector of the angle made by the segments that join $M$ to the foci), orthogonal to the bisector of the angle made by the rays that arrive at $M$ from $\vec{s}$ and $\vec{s}+\hat{h}(\vec{s})$, respectively (Fig. 29).

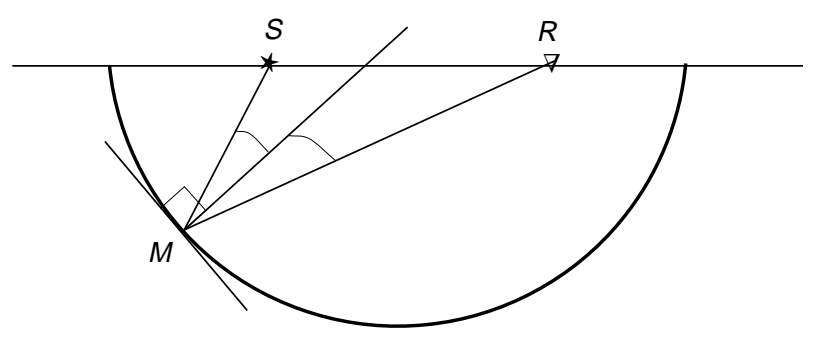

Figure 29

Migrated dip in 2D. At point $M$, the dip of the migrated event is the straight line tangent to the isochron, i.e. orthogonal to the bisector of the angle made by the rays that arrive at $M$ and originating in $S$ and $R$, respectively. 


\section{Partitioning of the Data by Offset Class}

Until now, we have considered a single offset class defined by function $\hat{h}(\vec{s})$. We are now going to consider a series (in fact a continuum) of offset classes and to introduce the adequate formalism.

\section{Case of a 2D Acquisition}

We start by setting the basis of the formalism in the $2 \mathrm{D}$ case which is much simpler to understand and which, in particular, allows graphical illustrations. Looking at Figure 30, offset classes can be understood as contour lines of some function $g(s, r)$ : the offset class $\lambda(\lambda \in \mathbb{R})$ is the set of source-receiver pairs such that $g(s, r)=\lambda$. We can readily realize that such a definition generalizes the notion of common offset gathers (we consider $g(s, r)=r-s$ ). Also, $\lambda$ being given, the associated offset class function $h_{\lambda}(s)$ is obtained from the definition of function $g$.

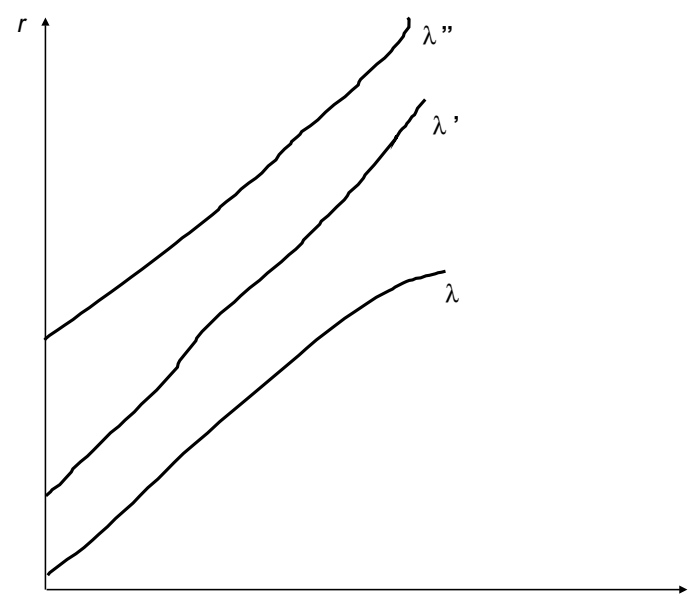

Figure 30

Three different offset classes. An offset class can be understood as the contour line of a function $g(s, r)$ : the offset class $\lambda$ is the set of source-receiver pairs such that $g(s, r)=\lambda$.

\section{Case of a 3D Acquisition}

In $3 \mathrm{D}$, a source-receiver pair consists in a vector in $\mathbb{R}^{4}$. The source location $\vec{s}$ being given, two data are required to obtain the two coordinates of the receiver location $\vec{r}$ in the offset class $\vec{\lambda}$. The following equation:

$$
\vec{g}(\vec{s}, \vec{r})=\vec{\lambda}
$$

involves now the equality between two vectors in $\mathbb{R}^{2}$.

We assume that, for $\vec{s}$ and $\vec{\lambda}$ given, Equation (15) admits a unique solution $\vec{r}$. This assumption makes the offset class function $h_{\vec{\lambda}}(\vec{s})$ single-valued. We can also notice that, the offset class being defined by means of the contour lines of some function $\vec{g}$, a source-receiver pair can belong to one offset class only. Finally, we will assume function $\vec{g}(\vec{s}, \vec{r})$ to be continuously differentiable and the Jacobian $\partial \vec{g} / \partial \vec{r}$ is never zero: this makes Equation (15) to admit a unique solution in $\vec{r}$, for $\vec{s}$ and $\vec{\lambda}$ given.

\section{Stack of Offset Class Migrated Images}

Considering the offset class associated with some $\vec{\lambda} \in \mathbb{R}^{2}$, we define the offset class migrated image at point $M$ by (this is nothing but a change in the notations as compared to Definition (10)):

$$
\begin{aligned}
& m(M, \vec{\lambda})= \\
& \int \mathrm{d} \omega i \omega f(\omega) \int_{S}\left[N(\vec{s}, \vec{\lambda}) e^{i \omega\left[t_{e}(\vec{s}, \vec{\lambda})-t_{m}(M ; \vec{s}, \vec{\lambda})\right]}\right] \mathrm{d}^{2} \vec{s}
\end{aligned}
$$

with $t_{e}$ the arrival time of the event for source $\vec{s}$ and receiver $\vec{r}, t_{m}$ the travel time from $\vec{s}$ to $M$ to $\vec{s}$ ( $\vec{r}$ denotes, for $\vec{s}$ and $\vec{\lambda}$ given, the receiver solution of Equation (15)), and:

$$
N(\vec{s}, \vec{r})=\left|\frac{\mathrm{d} \vec{q}}{\mathrm{~d} \vec{s}}\right| D_{e}(\vec{s}, \vec{r})
$$

where:

- $D_{e}(\vec{s}, \vec{r})$ is the specular scattering amplitude;

- $\left|\frac{\mathrm{d} \vec{q}}{\mathrm{~d} \vec{s}}\right|$ is the Jacobian of the map:

$$
\vec{s} \mapsto \vec{q}=\frac{\vec{s}+\vec{r}}{2}
$$

We have shown previously that the support of the offset class migrated event is localized at the vicinity of points $M$ such that there exists $\vec{s}$ so that the pair $(\vec{s}, M)$ is solution of the system:

$$
\left\{\begin{array}{c}
\Phi(M ; \vec{s}, \vec{\lambda})=0 \\
\frac{\partial \Phi}{\partial \vec{s}}(M ; \vec{s}, \vec{\lambda})=0
\end{array}\right.
$$

where $\Phi$ is defined by:

$$
\Phi(M ; \vec{s}, \vec{\lambda})=t_{e}(\vec{s}, \vec{\lambda})-t_{m}(M ; \vec{s}, \vec{\lambda})
$$

As acquisitions with vector $\vec{\lambda}$ depending continuously of two parameters and thus covering a domain of $\mathbb{R}^{2}$ are rarely available (we can hardly consider that we have data associated with arbitrary offset that is arbitrary norm and azimuth), we consider that vector $\vec{\lambda}$ ranges within a one-dimensional subset of $\mathbb{R}^{2}$ : we suppose that $\vec{\lambda}$ depends continuously of a scalar parameter $\alpha(\vec{\lambda}(\alpha)$ gives the parametric representation of the considered one-dimensional subset). 
The stack of offset class migrated images is then defined quite naturally: the value at point $M$ of the post-migration stack is given by:

$$
p(M)=\int m(M ; \vec{\lambda}(\alpha)) \mathrm{d} \alpha
$$

It is classical to evaluate Equation (16) by means of the stationary phase formula: the support of the event in the postmigration stack is localized at the vicinity of points $M$ such that there exists $(\vec{s}, \alpha)$ that makes the following system fulfilled:

$$
\left\{\begin{array}{l}
\Phi(M ; \vec{s}, \vec{\lambda}(\alpha))=0 \\
\frac{\partial \Phi}{\partial \vec{s}}(M ; \vec{s}, \vec{\lambda}(\alpha))=0 \\
\frac{\partial \Phi}{\partial \alpha}(M ; \vec{s}, \vec{\lambda}(\alpha))=0
\end{array}\right.
$$

The geometry of the event imaged in the post-migration stack thus appears as the envelope of the family (with generator $\alpha$ ) of offset class migrated events. Note also that Condition (17c) shows that the offset class migrated event appears horizontal when seen in the $\alpha$ direction (migration coherency panel) for $(M, \alpha)$ solution of System (17).

\section{Post-Migration Stack and Zero Offset}

The goal of this section is to show that, whatever the migration velocity model, the zero offset events constitute a subset of the events imaged in the post-migration stack. Namely we are going to show that if a pair $(\vec{s}, \alpha)$ is such that the unique receiver $\vec{r}$ solution of:

$$
\vec{g}(\vec{s}, \vec{r})=\vec{\lambda}(\alpha)
$$

coincides with $\vec{s}$ (we thus consider, in the offset class associated with $\alpha$, a trace, the one associated with $\vec{s}$, which is actually a zero offset trace), then the point $M$ imaged in the offset class migrated event (this point is defined once $\vec{s}$ is known) will be such that Equation (17c) is fulfilled or, in other words, the offset class migrated event will be part of the post-migration stack.

We thus consider, for $\vec{s}$ and $\alpha$ given, receiver $\vec{r}$ such that Equation (18) is fulfilled and $M$ solution of Equations (17a) and (17b). We give $\alpha$ a perturbation $\mathrm{d} \alpha, \vec{s}$ being unchanged. It results:

- a perturbation:
- and therefore a perturbation $\mathrm{d} \vec{r}$ solution of the system ([ $\partial \vec{g} / \partial \vec{r}]$ is a $2 \times 2$ matrix):

$$
\left[\frac{\partial \vec{g}}{\partial \vec{r}}\right] \mathrm{d} \vec{r}=\mathrm{d} \vec{\lambda}
$$

- and, hence, a perturbation:

$$
\mathrm{d} \Phi=\frac{\partial \Phi}{\partial \vec{\lambda}} \cdot \mathrm{d} \vec{\lambda}
$$

( $\cdot$ denotes the Euclidian scalar product between vectors $\partial \Phi / \partial \vec{\lambda}$ and $\mathrm{d} \vec{\lambda}$ in $\mathbb{R}^{2}$ ) of $\Phi(M ; \vec{s}, \vec{\lambda}(\alpha))$.

We are going to establish that $\mathrm{d} \Phi=0$ which will prove that Equation (17c) is fulfilled.

We introduce function $\Psi(M ; \vec{s}, \vec{r})$ defined by:

$$
\Psi(M ; \vec{s}, \vec{r})=\Phi(M ; \vec{s}, \vec{g}(\vec{s}, \vec{r}))
$$

Perturbation $\mathrm{d} \Phi$ is also equal to:

$$
\mathrm{d} \Phi=\frac{\partial \Psi}{\partial \vec{r}} \cdot \mathrm{d} \vec{r}
$$

with $\mathrm{d} \vec{r}$ solution of Equation (19).

If we now consider another perturbation in the sourcereceiver pair $\left(\mathrm{d} \vec{s}^{\prime}, \mathrm{d} \vec{r}^{\prime}\right)$ solution of the $4 \times 4$ system:

$$
\begin{aligned}
& {\left[\frac{\partial \vec{g}}{\partial \vec{s}}\right] \mathrm{d} \vec{s}^{\prime}+\left[\frac{\partial \vec{g}}{\partial \vec{r}}\right] \mathrm{d} \vec{r}^{\prime}=\overrightarrow{0}} \\
& \mathrm{~d} \vec{s}^{\prime}+\mathrm{d} \vec{r}^{\prime}=\mathrm{d} \vec{r}
\end{aligned}
$$

(this is a system in which $\partial \vec{g} / \partial \vec{s}$ and $\partial \vec{g} / \partial \vec{r}$ are $2 \times 2$ matrices; this system shows that perturbation $\left(\mathrm{d} \vec{s}^{\prime}, \mathrm{d} \vec{r}^{\prime}\right)$ does not perturb $\vec{\lambda}$ : we remain within the same offset class), then the resulting perturbation in $\Psi$ will be:

$$
\begin{array}{rlrl}
{\left[\frac{\partial \Psi}{\partial \vec{s}}\right] \mathrm{d} \vec{s}^{\prime}} & +\left[\frac{\partial \Psi}{\partial \vec{r}}\right] \mathrm{d} \vec{r}^{\prime} & \\
& =\left[\frac{\partial \Psi}{\partial \vec{r}}\right] \cdot\left(\mathrm{d} \vec{s}^{\prime}+\mathrm{d} \vec{r}^{\prime}\right) & \begin{array}{l}
\text { (consequence of the } \\
\text { reciprocity theorem) }
\end{array} \\
& =\left[\frac{\partial \Psi}{\partial \vec{r}}\right] \cdot \mathrm{d} \vec{r} & & (\text { from (21b)) } \\
& =\mathrm{d} \Phi & & (\text { from (20)) }
\end{array}
$$

As perturbation $\left(\mathrm{d} \vec{s}^{\prime}, \mathrm{d} \vec{r}^{\prime}\right)$ is a constant $\vec{\lambda}$ perturbation, $\mathrm{d} \Phi$ is zero as a consequence of Equation (17b). 